Review Article

\title{
Neuroprotective Role of the Nrf2 Pathway in Subarachnoid Haemorrhage and Its Therapeutic Potential
}

\author{
Ardalan Zolnourian $\mathbb{D}^{1,2}$ Ian Galea, ${ }^{1,2}$ and Diederik Bulters $\mathbb{D}^{1,2}$ \\ ${ }^{1}$ Department of Neurosurgery, Wessex Neurological Centre, University Hospital Southampton, Tremona Road, \\ Southampton SO16 6YD, UK \\ ${ }^{2}$ Clinical Neurosciences, Clinical \& Experimental Sciences, Faculty of Medicine, University of Southampton, Tremona Road, \\ Southampton SO16 6YD, UK
}

Correspondence should be addressed to Ardalan Zolnourian; z.ardalan@gmail.com

Received 27 November 2018; Revised 17 February 2019; Accepted 20 March 2019; Published 2 May 2019

Academic Editor: Cristina Angeloni

Copyright (c) 2019 Ardalan Zolnourian et al. This is an open access article distributed under the Creative Commons Attribution License, which permits unrestricted use, distribution, and reproduction in any medium, provided the original work is properly cited.

\begin{abstract}
The mechanisms underlying poor outcome following subarachnoid haemorrhage (SAH) are complex and multifactorial. They include early brain injury, spreading depolarisation, inflammation, oxidative stress, macroscopic cerebral vasospasm, and microcirculatory disturbances. Nrf2 is a global promoter of the antioxidant and anti-inflammatory response and has potential protective effects against all of these mechanisms. It has been shown to be upregulated after SAH, and Nrf2 knockout animals have poorer functional and behavioural outcomes after SAH. There are many agents known to activate the Nrf2 pathway. Of these, the actions of sulforaphane, curcumin, astaxanthin, lycopene, tert-butylhydroquinone, dimethyl fumarate, melatonin, and erythropoietin have been studied in SAH models. This review details the different mechanisms of injury after SAH including the contribution of haemoglobin $(\mathrm{Hb})$ and its breakdown products. It then summarises the evidence that the Nrf2 pathway is active and protective after SAH and finally examines the evidence supporting Nrf2 upregulation as a therapy after SAH.
\end{abstract}

\section{Introduction}

SAH is a devastating condition and is associated with high levels of morbidity and mortality [1]. Despite advances in treatment, $40 \%$ of SAH survivors remain dependant due to physical disability, behavioural, and cognitive disturbances [2-4] and even amongst those who are seemingly independent, $50 \%$ suffer from neurocognitive deficits [5].

The mechanisms leading to poor outcome after SAH are complex and multifactorial. They include early brain injury [6] and a host of different ensuing processes including oxidative stress [7], inflammation [8], spreading depolarisation [9], and microscopic [10] and macroscopic vasospasm [11].

The purpose of this review is to evaluate the evidence for pharmacological augmentation of the Nrf2 pathway as a treatment for patients with SAH. In order to do this, we have first reviewed the mechanisms underlying poor outcomes after SAH, then reviewed the Nrf2 pathway, before considering how the Nrf2 pathway applies to SAH, and finally reviewed the evidence for specific compounds known to upregulate Nrf2 activity.

\section{Mechanisms of Injury after SAH}

The mechanisms leading to poor outcome after SAH can be categorised into primary and secondary injury in a way analogous to head injury, where the immediate damage that occurs directly from the insult is classed as primary and any further subsequent indirect damage as a result of the processes initiated by the insult is secondary. The important clinical distinction is the presumption that secondary injury is potentially treatable, whereas primary injury is not. While this concept is well established in head injury, the terms are not as widespread in $\mathrm{SAH}$ where the immediate injury is often classed together with subsequent events in the first 72 hours as early brain injury, and all ensuing events are considered separately. The latter events could also be grouped together as delayed brain injury. While some 
mechanisms like cerebral vasospasm clearly follow this classification, there are limitations, and others such as spreading depolarisation straddle both time periods.

2.1. Early Brain Injury. At the time of SAH, intracranial pressure rises to that of diastolic arterial pressure or higher $[12,13]$. This will in turn result in reduction of cerebral blood flow and cerebral perfusion pressure [14-16]. Consequently, cerebral autoregulation is disturbed [17, 18]. Blood-brain barrier (BBB) dysfunction [19], cerebral oedema [20, 21], and neuronal cell death [22] all take place within 72 hours from injury. Altered ionic homeostasis, excitotoxicity, thrombin activation [23], vascular integrity degradation [24], oxidative stress [7], inflammation [8], elevated matrix metalloproteinase-9 (MMP9) [25], and activation of the nitric oxide synthase (NOS) pathway $[26,27]$ are all seen.

2.2. Cerebral Vasospasm. Following lysis of red blood cells in the subarachnoid space, the central nervous system is exposed to high levels of $\mathrm{Hb}$ and its degradation products which lead to narrowing of the cerebral vessels and development of delayed cerebral ischaemia (DCI) in 30\% of patients $[11,28]$. In addition, DCI is diagnosed clinically in patients with reduced consciousness state and/or neurological deficits, the exact cause of which remains unclear. It is, however, part of the secondary brain injury and is most likely dependent on the initial pathological process after SAH.

Following $\mathrm{SAH}$, oxyhaemoglobin $(\mathrm{OxyHb})$ has been shown to induce vasoconstriction in animal models [29-31]. $\mathrm{OxyHb}$ is thought to cause arterial contraction directly and via the production of reactive oxygen species (ROS), since both $\mathrm{Hb}$ and ROS scavenge free nitric oxide [32]. OxyHb also decreases the activation of $\mathrm{K}^{+}$channels which leads to an intracellular surge of calcium and promotes vasoconstriction $[32,33]$. However, the exact pathophysiology remains unknown. The underlying mechanisms are thought to be multiple [34] and include oxidative stress [35], neuronal apoptosis $[36,37]$, decreased production of nitric oxide $[6,26]$, increased endothelin-1 [38, 39], calcium [40, 41], prostaglandin [42], thromboxane levels [32, 43], and spreading cortical depolarisation $[9,44]$. This ultimately results in cerebral ischaemia peaking between days 4 and 14 postictus [11, 45]. Regardless of the predominating mechanism, there is a clear relationship between cerebral vasospasm and the amount of subarachnoid blood [46, 47].

However, macrovascular vasospasm does not always correlate directly with the development of DCI. In fact, transcranial Doppler and angiographic studies have only shown a positive predictive value of $57 \%$ and $76 \%$, respectively [48]. When combined together, this is as high as $67 \%$ [49]. The degree to which macroscopic vasospasm influences outcome was further put in doubt by studies of the endothelin receptor antagonist, clazosentan, which demonstrated large consistent reductions in angiographic vasospasm without associated improvement in clinical outcomes [50].

It has therefore been proposed that poor outcome is conferred by spasm of the microvasculature rather than macroscopic vasospasm seen on angiography [51]. Spasm of arterioles has been shown in animal models of $\mathrm{SAH}$
[52-54]. In a mouse SAH model, vasoconstriction of arterioles was seen in more than $70 \%$ of subjects starting at three hours and persisting for at least three days after haemorrhage. An inverse correlation between the size of the arterioles and the extent of vasoconstriction was observed. In addition, $30 \%$ of the arterioles were occluded by microthrombi. The vessels were more likely to be severely constricted if there was already evidence of microthrombi. These findings may explain the poor cerebral perfusion pressure which may lead to DCI after SAH [55]. In a dog SAH model, morphometric examination of the internal diameter of arterioles revealed significant reduction, with marked increase in vessel wall thickness three and seven days after SAH [53].

An intraoperative study used orthogonal polarisation spectral imaging during aneurysm surgery to visualise the response of the small cortical vessels to hypercapnia. Patients with visible blood clot who underwent early surgery had a more pronounced vasoconstrictive response (39\%) compared to the group without visible blood clot who had late surgery (17\%) and patients with unruptured aneurysms (7\%) [10]. In addition, microvascular vasospasm in patients with DCI has been demonstrated by measuring the cerebral circulation time from digital subtraction angiograms. Prolonged cerebral circulation time as a marker of microvascular vasospasm was shown to be directly related to decreased regional cerebral blood flow [56].

These findings are consistent with a postmortem study of 53 aneurysmal SAH patients which demonstrated extensive cortical and hypothalamic infarctions with histologic evidence of microangiopathy [57]. Small cortical and hypothalamic infarcts have been noted in other postmortem studies [56]. The relationship between microvascular vasospasm and DCI may also explain why up to $25 \%$ of patients have CT evidence of infarction in a different location to the spastic artery or have no evidence of macrovascular spasm $[58,59]$.

2.3. Oxidative Stress. After $\mathrm{SAH}$, free extracellular $\mathrm{Hb}$ undergoes oxidation to methaemoglobin (MetHb), which then degrades into haem. Free haem is toxic and acts as a catalyst for formation of ROS causing oxidative stress. Haem toxicity is exerted by its proinflammatory properties as well as damage caused by ROS leading to modification of lipids, carbohydrates, and nucleotides with eventual cell death affecting both neurons [60] and endothelial cells [61].

Following conversion of $\mathrm{OxyHb}$ to $\mathrm{MetHb}$, superoxide radicals are released which convert to hydroxyl radicals [31]. Human studies show evidence of an increase in oxidative stress and lipid peroxidation in both CSF and serum as early as three days after SAH [7, 62]. These increases are more commonly seen in patients with poor outcome [63]. Blocking lipid peroxidation with a non-glucocorticoid aminosteroid, tirilazad, has been assessed in five randomised clinical trials. However, meta-analysis of these trials showed no improvement in clinical outcomes in patients [64].

ROS produce vasoactive lipids via reactions with arachidonic acid, resulting in vasoconstriction. Furthermore, free radical oxidation of bilirubin and biliverdin leads to formation of bilirubin oxidation products [65]. The accumulation 
of these products and bilirubin in the CSF has been shown to be associated with DCI and vasospasm after SAH [66].

Oxidative stress has been linked to the activation of protein kinase $C[6,67]$ and Rho kinase $[6]$, both of which are involved in smooth muscle contraction. Protein kinase C plays an important role in vascular smooth muscle cell growth as well as vascular remodelling, as seen in vasospasm [32]. Intra-arterial fasudil (a Rho kinase inhibitor) has been successful in experimental SAH [68], and it has also been used in patients reducing average arterial circulation time with potential reduction in cerebral vasospasm [69]. However, the clinical benefit of this remains uncertain.

2.4. Inflammation. Following $\mathrm{SAH}$, free $\mathrm{Hb}$ released in the subarachnoid space stimulates rapid expression of cell adhesion molecules by endothelial cells, attracting neutrophils [1]. These cells are subsequently trapped in the subarachnoid space and may be implicated in vasospasm through the enzymatic activity associated with the oxidative burst. Constant inflammatory stimulation may result in chronic inflammation involving lymphocytes and monocytes [70]. Monocytes invade the injured tissue to become macrophages. Lymphocytes and macrophages release inflammatory cytokines including IL- $1 \beta$, IL- 6 , and TNF- $\alpha$ in the CSF [71]. Bloodbrain barrier breakdown further accentuates release of inflammatory cytokines, which peaks at day seven postictus. Increasing levels of IL-1, IL- 6 and TNF- $\alpha$ have been shown to be associated with poor outcome [72-75], and blockade with interleukin-1 receptor antagonist (IL-1RA) has been shown to reduce this [76-78]. Inflammation in the brain is also linked to post-SAH systemic inflammatory response syndrome and organ failure [1]. Neuroinflammation has been shown to be associated with cognitive dysfunction in other disorders $[79,80]$ so this could be a putative mechanism underlying such deficits in SAH patients.

2.5. Cortical Spreading Depolarisation. Cortical spreading depolarisation (CSD) refers to slow waves of near-total neural depolarisation with resultant cellular swelling due to the influx of cations across the cell membrane. This exceeds the ATP-dependent $\mathrm{Na}^{+}$and $\mathrm{Ca}^{2+}$ pump activity which leads to shrinkage of the extracellular space due to water influx [9]. CSD can be induced by a variety of means [81]. It can occur immediately after SAH and even up to two weeks from the cerebral insult [82]. After SAH, it is usually triggered by high $\mathrm{K}^{+}[83,84]$ released from degraded erythrocytes or alternatively from cortical injury from the initial bleed.

The normal response to a short episode of CSD is hyperaemia. However, in SAH following a single wave of CSD associated with $\mathrm{OxyHb}[85,86]$, reduced nitric oxide concentration [83, 87], or endothelin-1 [88], the normal response is reversed [89]. Moreover, CSD triggers vasoconstriction resulting in cortical spreading ischaemia (CSI) $[85,90]$ which may lead to cortical necrosis [86]. In addition, prolonged or repetitive CSD can lead to tissue damage without CSI, simply by the increased metabolic demand, i.e., increased oxygen utilisation [91].

As well as in animal studies [84-86, 92, 93], CSD and CSI have been demonstrated in SAH patients $[9,44,94]$. The multicentre Co-Operative Studies on Brain Injury Depolarizations (COSBID) showed a strong association between CSD and DCI for the first time in humans. 13/18 patients (72\%) demonstrated signs of CSD on electrocorticography recorded via a subdural strip over the cerebral cortex that was placed during the craniotomy and was monitored for ten days. Seven patients developed DCI. CSD had positive and negative predictive values of $86 \%$ and $100 \%$, respectively. This study also revealed that DCI may occur in the absence of radiological vasospasm but is still associated with clusters of spreading depolarisation, meaning that large vessel spasm is not the main driver of DCI [44]. Moreover, spreading depolarisation upregulates multiple genes, such as haemoxygenase-1 (HO-1) [95], which could be protective and might explain why in some patients subsequent ischaemia does not take place.

\section{Nuclear Factor-Erythroid 2- (NF-E2-) Related Factor 2 (Nrf2)}

Nuclear factor-erythroid 2- (NF-E2-) related factor 2 (Nrf2) is a redox-sensitive transcription factor belonging to the cap'n'collar (CNC) subclass of the basic leucine zipper region containing the protein family. It binds to a specific DNA site, the antioxidant response element (ARE), regulating transcription of an array of detoxifying or antioxidant enzymes. These include gamma-glutamylcysteine synthetase, superoxide dismutase, catalase, glutathione reductase, thioredoxin reductase, peroxiredoxins, and glutathione S-transferase (GST- $\alpha 1)[96,97]$. It also regulates degradation of red blood cells, $\mathrm{Hb}$, haem, and iron through transcriptional upregulation of CD36 [98], haptoglobin (Hp) [99], haemopexin [100], HO-1 [101], and ferritin [102].

$\mathrm{Hp}$ has received particular attention following SAH. It is the fourth most abundant plasma protein and is synthesised in the liver and reticuloendothelial system [103]. Outside the brain, extracellular $\mathrm{Hb}$ is immediately bound to $\mathrm{Hp}$ via an extremely strong interaction [104]. Hp is not synthesised in the brain under normal physiological conditions and diffuses into the CNS. In the pathologic state, it is expressed by astrocytes [105] and oligodendrocytes [99]. The Hb-Hp complex is recognised by the CD163 membrane receptor, leading to internalisation [106]. In the CSF, concentration of $\mathrm{Hp}$ is two orders of magnitude lower than in the circulation, which is insufficient to bind all $\mathrm{Hb}$ arising from the clot [107]. The CD163 uptake system is saturated, as evidenced by the presence of the Hb-Hp complex in the CSF after SAH [107].

Although no suitably powered human study of CSF Hp levels has been done to assess its relationship with outcome, the Hp phenotype has been shown to be important in determining outcome after SAH. Human Hp is composed of two peptide chains: $\alpha$ and $\beta$. There are two different $\alpha$ alleles giving rise to three different phenotypes: $\alpha 1 \alpha 1, \alpha 1 \alpha 2$, and $\alpha 2 \alpha 2$ [108]. The Hp $\alpha 2$ genotype has been shown to be associated with cerebral vasospasm [109-112], cerebral salt wasting [113], and poor outcome $[113,114]$. In addition to the Hp-dependent pathway, there are other less efficient scavenging systems such as cubilin and megalin [115] and 


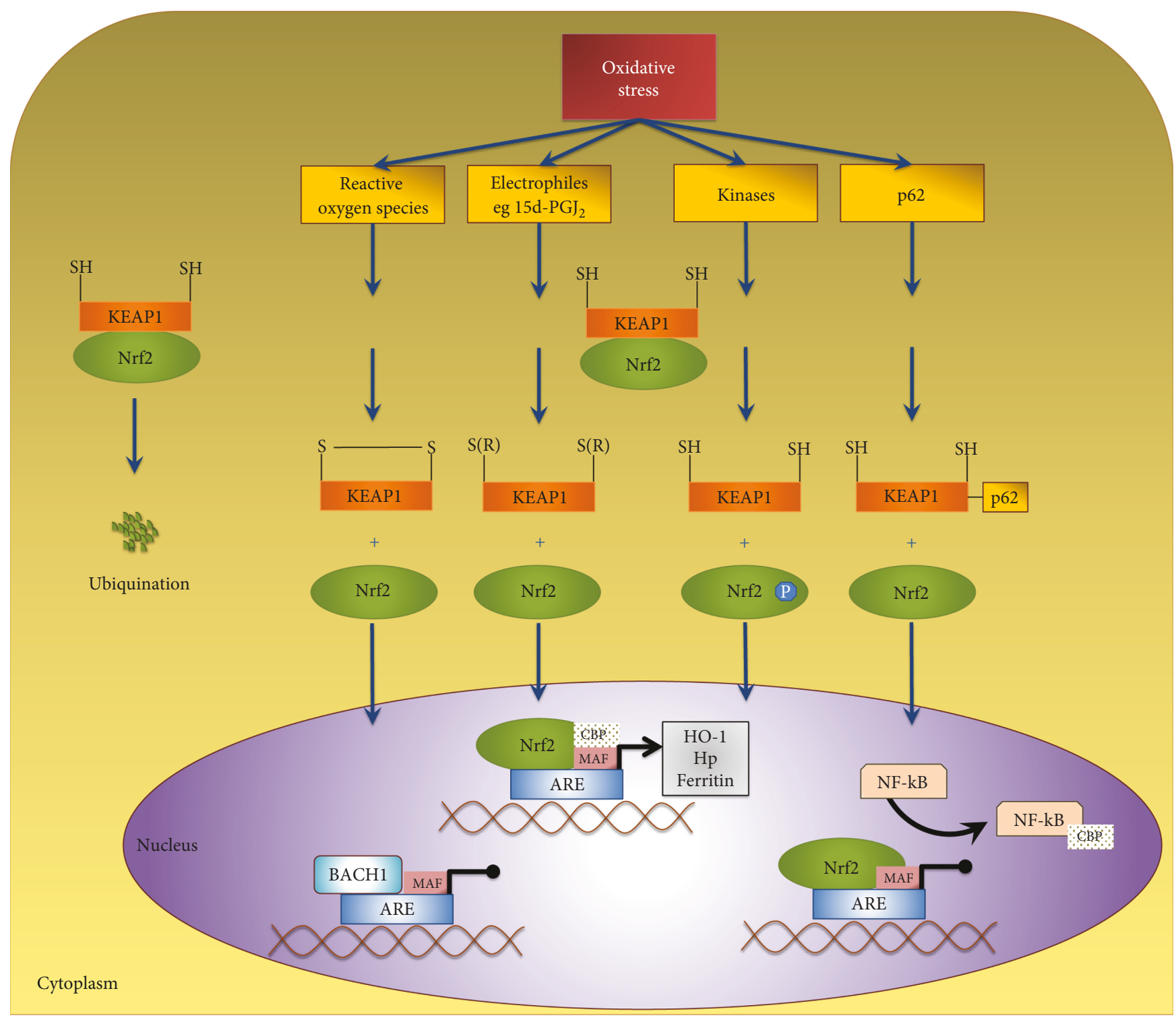

FIGURE 1: Nrf2 regulation. Nrf2 is a redox-sensitive transcription factor that is bound to KEAP1 under physiological condition. KEAP1 is an intracellular redox sensor and targets Nrf2 for ubiquitination. Following oxidative stress, four different mechanisms result in dissociation of KEAP1 from Nrf2. These four mechanisms are as displayed in order: (1) oxidation of cysteine residues by lower molecular weight reactive oxygen species, (2) covalent modification of cysteine residues by electrophiles such as NF- $\kappa \mathrm{B}$-induced cyclopentenone prostaglandins, (3) phosphorylation of Nrf2 at Ser40 by protein kinase C and PERK, and (4) protein-protein interaction between p62 and KEAP1. Free of KEAP1, Nrf2 translocates into the nucleus where it binds to antioxidant response elements in DNA to mediate transcription of key proteins. Nrf2 requires the binding partners MAF and $\mathrm{CBP}$ to initiate transcription. BACH1 competes for MAF and NF- $\kappa \mathrm{B}$ competes for CBP. Overall, the equilibrium between the two transcriptions factors BACH1 and Nrf2 determines overall transcription of the downstream genes.

downstream haem clearance via the haemopexin-CD91 pathway [116].

3.1. Nrf2 Regulation. During normal physiological conditions, Nrf2 is bound to the Kelch-like ECH-associated protein 1 (KEAP1) in the cytoplasm [96] (Figure 1). KEAP1 is a homodimer with three major domains, one of which facilitates ubiquitination of Nrf2 [117]. KEAP1 is an intracellular redox sensor. In response to oxidative stress as happens after SAH, key cysteine residues on KEAP1 are oxidised [117]; in addition, Nrf2 is phosphorylated on Ser40 by protein kinases
[118-120]. One or both events lead to Nrf2 release from KEAP1; Nrf2 then translocates into the nucleus, to act as a transcription factor [121] (Figure 1). Furthermore, p62 is a protein that has six domains, one of which is the KEAP1-interacting region (KIR) [122-124]. The protein p62 has been identified to be involved in activation of Nrf2 $[124,125]$ by inhibiting KEAP1-mediated Nrf2 ubiquitination, leading to stabilisation and a rise in Nrf2 levels $[123,124]$.

While KEAP1, as an intracellular redox sensor, regulates the transcriptional response to oxidative stress through $\mathrm{Nrf2}$, 
this is balanced by the activity of other transcription factors such as nuclear factor kappa-light-chain-enhancer of activated $\mathrm{B}$ cells $(\mathrm{NF}-\kappa \mathrm{B})$ and $\mathrm{BTB}$ (broad complex, tramtrack, bric-a-brac) and $\mathrm{CNC}$ homology 1 (BACH1). NF- $\kappa \mathrm{B}$ and Nrf2 both need to bind CREB-binding protein (CBP) to exert their transcriptional effects, so activation of NF- $\kappa \mathrm{B}$ can inhibit the Nrf2 transcriptional programme by limiting the availability of CBP [126] (Figure 1). BACH1 competes with Nrf2 to bind to ARE and can repress the effects of Nrf2 [127]. It is the equilibrium between $\mathrm{Nrf} 2$ and $\mathrm{BACH} 1$ that determines the production of genes under their control (Figure 1).

3.2. Nrf2 in the Brain. Nrf2 is expressed in the central nervous system (CNS), and it is upregulated in response to inflammation and cerebral insults [128]. Nrf2 therefore plays a key role in conditions where inflammation is the hallmark. For example, both ischaemic and haemorrhagic strokes share some common pathophysiologic pathways with SAH. Outcomes after both have been shown to be ameliorated by activation of the Nrf2 pathway.

A number of studies of ischaemic stroke models [129-131] have shown that Nrf2 levels rise soon after the onset of stroke. In a permanent ischaemic stroke model, this was as early as three hours from the ictus and peaked at 24 hours postinsult [132]. In a transient stroke model, a significant increase in $\mathrm{Nrf} 2$ was seen at two hours with a peak at eight hours postreperfusion and decreasing at 24 hours. Nrf2 levels were measured in both the peri-infarct and the core ischaemic regions with a marked increase in the peri-infarct area [129]. Increased levels of peri-infarct Nrf2 is most likely due to the increased oxidative stress in this region [129]. Furthermore, Nrf2 expression has been demonstrated in neurons, astrocytes, leukocytes, and microglia [129, 130, 132]. Nrf2 knockout models are also associated with poorer neurological recovery $[133,134]$.

The Nrf2 pathway is also activated following intracerebral haemorrhage $(\mathrm{ICH})$ in mice. $\mathrm{HO}-1$ was shown to be upregulated after 24 hours, peaking at five days with a return to baseline on day eight [101]. Nrf2 knockout mice suffered more severe neurological deficits. There was larger injury volume, increase in leukocyte infiltration, production of ROS, DNA damage, and cytochrome c release during the critical early phase of the post-ICH period with poorer neurological recovery [135].

Nrf2 is also protective against hemin toxicity. Rat astrocyte cultures were pretreated with $\mathrm{Hb}$ or vehicle, and then exposed to hemin, the degradation product of $\mathrm{Hb}$. Pretreated astrocytes showed resistance to toxicity induced by hemin. Pretreatment with $\mathrm{Hb}$ was shown to induce Nrf2 and $\mathrm{HO}-1$, and the latter led to haem catabolism, in keeping with the protective effect of $\mathrm{Hb}$ pretreatment. In support of this mechanism, the protective effect of $\mathrm{Hb}$ pretreatment was lost in Nrf2 knockdown cells [136].

3.3. Nrf2 in SAH. Experimental data have shown that Nrf2 expression is upregulated in the basilar artery of rats after SAH [137]. This was observed in the nucleus and cytoplasm of endothelial cells, smooth muscle cells, and adventitial cells on day five following SAH, demonstrating marked activation of the Nrf2 system [137].

Chen and colleagues using a rat SAH model demonstrated that Nrf2 expression is also increased in the cortex at 12 hours, 24 hours, and 48 hours postinjection of blood compared to controls, with a peak at 24 hours postinjection [138].

Deletion of Nrf2 has been shown to be associated in vitro with an increased inflammatory response in cultured murine astrocytes. In a model utilising primary cultured astrocytes exposed to $\mathrm{OxyHb}$, downstream inflammatory cytokines such as TNF- $\alpha$, IL- $1 \beta$, and IL- 6 as well as MMP9 were significantly higher in Nrf 2 knockout mice, and this was accompanied by $\mathrm{NF}-\kappa \mathrm{B}$ upregulation [139]. The effect of Nrf2 knockout was also studied in an in vivo mouse SAH model. Brain oedema and neural cell death after SAH were measured and compared between wild-type mice and Nrf2 knockout mice. Nrf2 deficiency increased brain oedema and neural cell death at 24 hours after SAH. Neurological deficits as measured by posture, grooming, and ambulation were also markedly worse in Nrf2-deficient mice [140].

The pathophysiology of SAH involves oxidative stress and inflammation. The redox state can be assessed using malondialdehyde (MDA) levels and the GSH/GSSG ratio. MDA is a lipid peroxidation product and is elevated after oxidative stress [141]. The GSH/GSSG ratio is thought to represent antioxidative capacity and is decreased in many inflammatory CNS disorders [142, 143]. Nrf2 knockout mice were found to have higher MDA levels and a lower GSH/GSSG ratio. Furthermore, inflammatory cytokines including TNF- $\alpha$ and IL- $1 \beta$ were significantly increased [140]. Cerebral vasospasm at 24 hours after experimental $\mathrm{SAH}$ in Nrf2 knockout mice was not significantly different from wild-type animals. This could suggest that cerebral vasospasm may occur independently of inflammation and oxidative stress. However, another study showed that Nrf2 upregulation was associated with a decreased rate of vasospasm in a rat model of SAH [144]. It is possible that the different results in vasospasm observed between these two studies are due to compensatory mechanisms in the Nrf2 knockout mice, or other technical differences.

\section{Therapeutic Potential of Nrf2 Activators in SAH}

There are a large number of known activators of the Nrf2 system. All act by binding KEAP1 releasing Nrf2, which translocates to the nucleus leading to increased transcription. Nrf2 activators are broadly classified as electrophilic cysteine-reactive compounds and nonelectrophilic Keap1-Nrf2 protein-protein interaction inhibitors. Most well-established compounds fall into the former category. However, many are pleiotropic and their primary mechanism of action remains controversial. There are also efforts being made to develop new more selective compounds of the latter category. These have the potential to be more potent inducers with less cross-activation of other pathways [145].

SAH represents an ideal condition for these treatments. With the wide range of proteins upregulated by $\mathrm{Nrf} 2$, it 
can be postulated that Nrf2 activation may have beneficial effects on any of the described secondary mechanisms underlying poor outcome. The magnitude of this effect is likely to be dictated by the timing of administration of the Nrf2 activator.

Following SAH, DCI occurs no earlier than three days after the event and is not seen beyond 21 days. Even if the mechanisms leading to it are initiated earlier, Nrf2 preconditioning has been shown to be protective in ischaemic stroke. Therefore, a case can be made for administration as late as 72 hours after SAH, which would be consistent with most previous drug studies in SAH [146].

Prevention may require earlier treatment. Most discussed mechanisms are either initiated or worsened by Hb. Given that it takes days for red cell lysis, and intracellular $\mathrm{Hb}$ to be released, CSF $\mathrm{Hb}$ levels progressively rise from day one to day six after SAH [147]. This also offers a therapeutic window during which the Nrf2 system can be preemptively fully induced to ameliorate this. This is likely to have effects on macro- and microvascular spasm and $\mathrm{Hb}$-mediated components of oxidative stress and inflammation.

However, other aspects of inflammation and oxidative stress may result directly from early brain injury. These and indeed early brain injury itself would require earlier treatment still. Preconditioning would offer the greatest chance of benefit but is clearly not practical.

Therefore, aiming for treatment in patients at the earliest available opportunity, but accepting treatment up to 72 hours after SAH where patients do not present immediately, would seem a pragmatic approach, although early phase studies may benefit from shorter recruitment windows to increase the chance of observing an effect. Here, we have reviewed all agents that have been tested and have shown therapeutic potential in SAH. We have summarised the characteristics of each Nrf2 activators in Table 1 and listed the individual animal studies of Nrf2 activators in experimental SAH in Table 2.

\section{Sulforaphane}

Sulforaphane (SFN), 1-isothiocyanate-(4R)-(methylsulfinyl) butane, is a widely studied isothiocyanate. SFN stabilizes Nrf2 by inhibiting its ubiquitination. Oxidation of critical cysteine residues of KEAP1 by SFN appears to be essential [117], but subsequent mechanistic steps are a matter of controversy. While it has been widely believed that Nrf2 stabilisation occurs by freeing Nrf2 from KEAP1, as happens naturally when KEAP1 cysteines are oxidised, this has been recently challenged with a suggestion that Nrf2 is stabilised in complex with KEAP1 in the nucleus [148]. Although Nrf2 phosphorylation at Ser40 via protein kinase pathways may be involved in its stabilisation during oxidative stress [118-120], this is not implicated in chemically induced stabilisation of Nrf2 by SFN [148]. In line with stabilisation of $\mathrm{Nrf2}$, SFN leads to upregulation of $\mathrm{Hp}$ expression in the periphery [149] and in the brain [99].

The effect of SFN has been assessed in an in vitro $\mathrm{SAH}$ model. Rat aortic arch cells were exposed to OxyHb and SFN for 48 hours. Levels of Nrf2 and Nrf2-regulated genes including $\mathrm{HO}-1$ and NQO1 (NAD(P)H:quinone oxidoreductase 1) were significantly increased and further upregulated when exposed to SFN. In addition, the concentrations of inflammatory cytokines IL- $1 \beta$, IL-6, and TNF- $\alpha$ were markedly reduced in the SFN group [150].

The effects of SFN after SAH in vivo were first assessed by Chen et al. [138]. Autologous blood was injected in the prechiasmatic cistern of rats. Intraperitoneal SFN was injected at 30 minutes, 12 hours, and 36 hours. mRNA expressions of HO- 1 , NQO1, and GST- $\alpha 1$ were measured in the cortex of the animals after 48 hours. $\mathrm{SAH}$ led to increased expression of HO-1, NQO1, and GST- $\alpha 1$ in the rat cortex. A further significant increase was seen after treatment with SFN demonstrating that SFN has the capacity to increase Nrf2 activity even in an already highly induced state. Brain oedema, BBB permeability, and apoptotic cell death were all reduced following treatment with SFN. Treatment with SFN was associated with a reduction in motor deficits in the rotarod test performed at 24 hours. These results demonstrate that SFN upregulates the Nrf2-ARE pathway after SAH and reduces early brain injury. It was associated with improved early function, although further study would be needed to demonstrate if this translates to better long-term outcomes [138].

More recently, an experimental study [144] assessed the effects of SFN on cerebral vasospasm after SAH. Autologous blood was injected in the cisterna magna of rats and repeated after 48 hours. Intraperitoneal injection of SFN was then performed every 24 hours from 30 minutes after induction of SAH to the third and last day of the experiment. Tissues were harvested three days after SAH. Cross-sectional areas of basilar arteries showed a significant difference between the SFN and untreated SAH groups. SFN administration increased the mRNA expression levels of Nrf2, HO-1, and NQO1 as well as significantly upregulating Nrf2 in endothelial and smooth muscle cells. The inflammatory cytokines IL$1 \beta$, IL-6, and TNF- $\alpha$ were significantly reduced following treatment with SFN. SFN was also found to ameliorate the behavioural deficits of rats following $\mathrm{SAH}$ as demonstrated by improvement in appetite and activity scores, but this was only performed once at three days following SAH. Overall, these results confirm that early administration of SFN upregulates the Nrf2 pathway after SAH, reduces vasospasm, and improves function at least early after SAH [144].

In addition to reducing oxidative stress in the subarachnoid space and consequently reducing cerebral vasospasm, SFN may have a beneficial effect on the ischaemia which can follow cerebral vasospasm. In a rat middle cerebral artery (MCA) occlusion, stroke model preconditioning with SFN one hour prior to stroke and reperfusion after four, 24, and 72 hours upregulated Nrf2 and $\mathrm{HO}-1$ expression leading to attenuation of $\mathrm{BBB}$ disruption, lesion progression as assessed by magnetic resonance imaging between 24 and 72 hours, and neurological dysfunction (based on motility, grasping reflex, and placing reaction) [151]. In a rat ischaemic stroke model, SFN was shown to reduce infarct volume following temporary occlusion of the left common carotid artery or MCA. Animals in the treatment group were injected with intraperitoneal SFN 15 minutes after the onset of ischaemia. SFN was found to increase brain HO-1 mRNA. The overall infarct volume was reduced in the treated group by $30 \%$ [152]. 


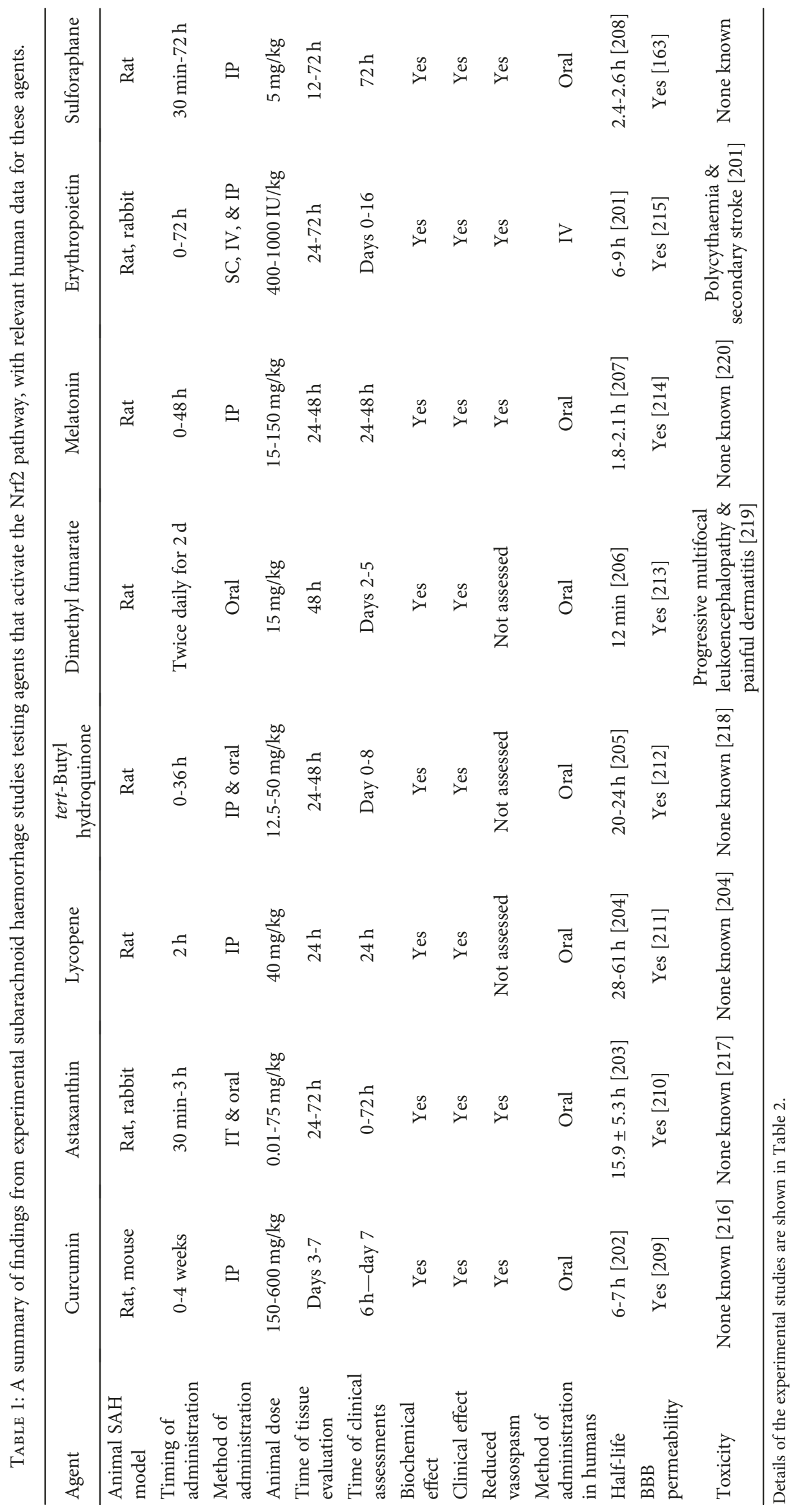




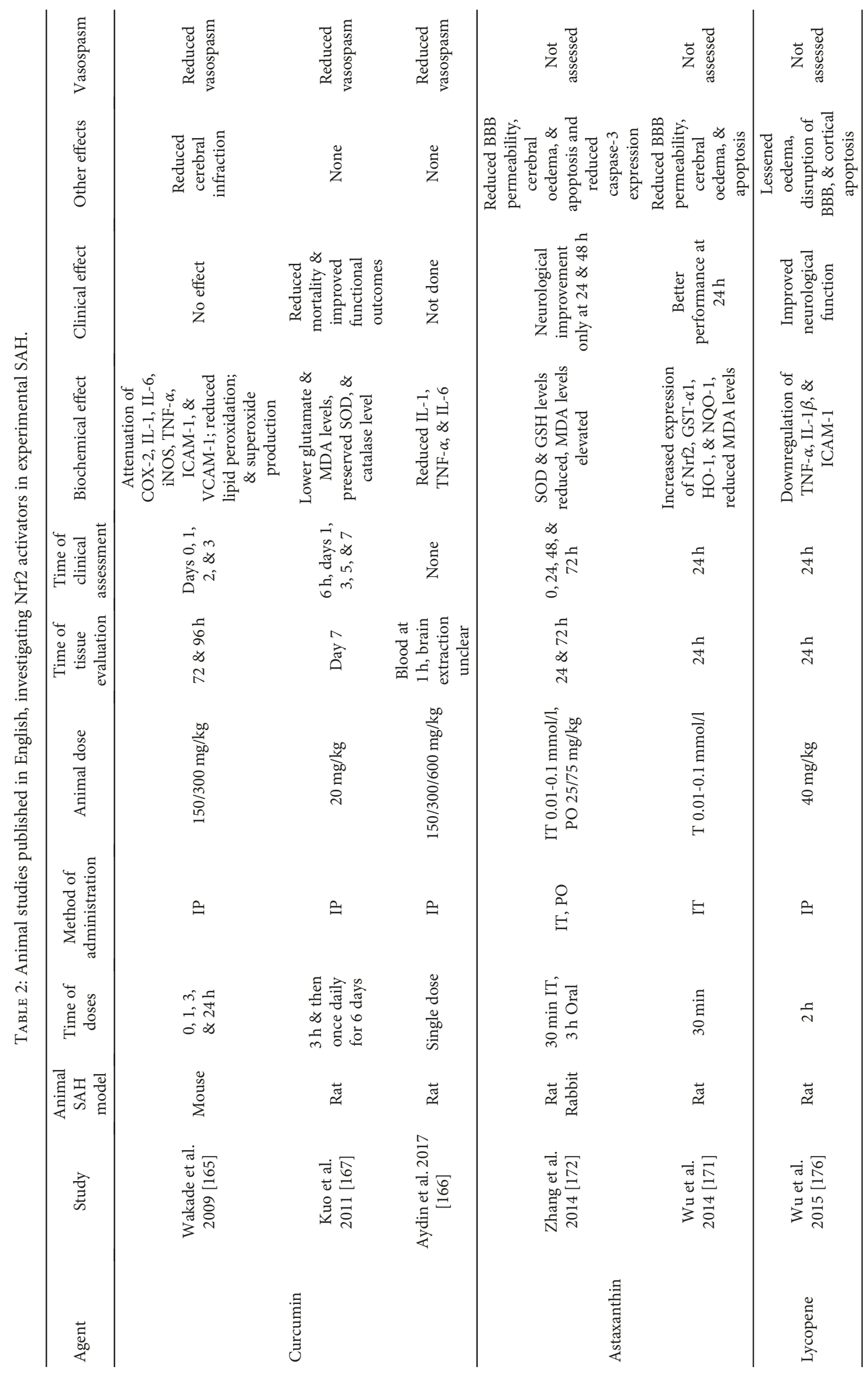




\begin{tabular}{|c|c|c|c|c|c|c|c|c|}
\hline 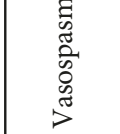 & 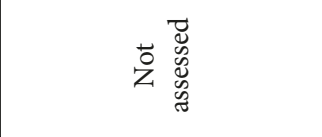 & 苍蒡 & 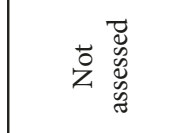 & 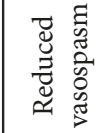 & 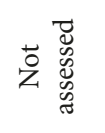 & 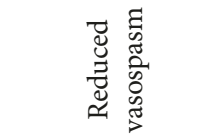 & 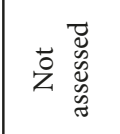 & 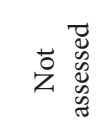 \\
\hline 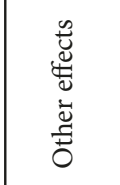 & 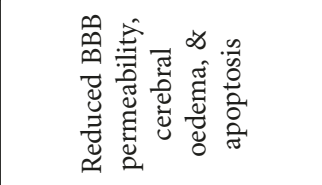 & 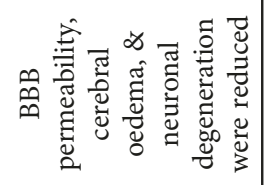 & 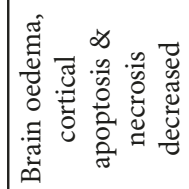 & 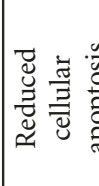 & 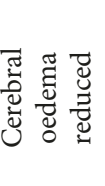 & 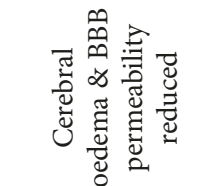 & 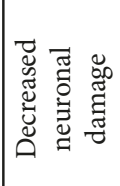 & $\begin{array}{l}\tilde{0} \\
\check{z}\end{array}$ \\
\hline 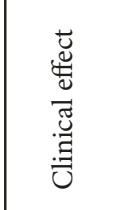 & 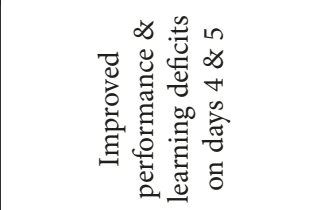 & 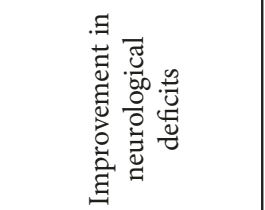 & 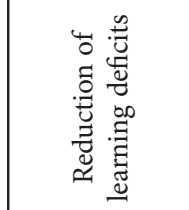 & 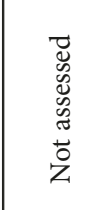 & 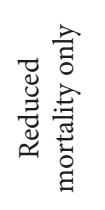 & 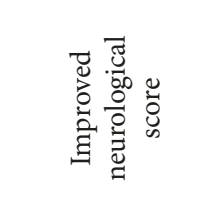 & 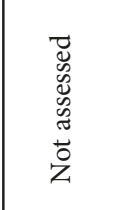 & 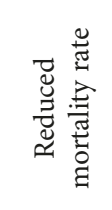 \\
\hline 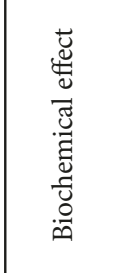 & 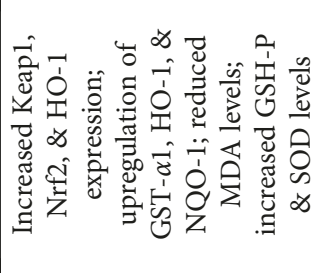 & 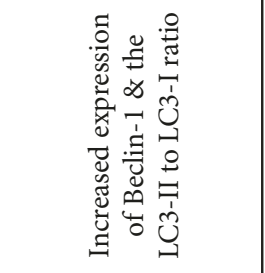 & 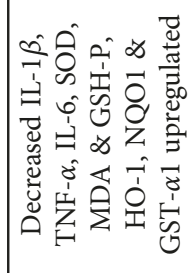 & 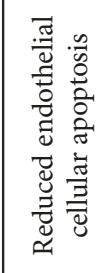 & 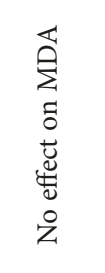 & 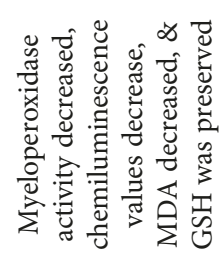 & 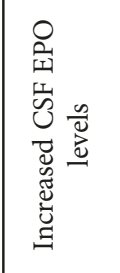 & 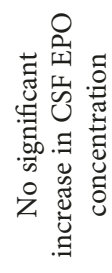 \\
\hline 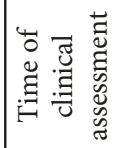 & 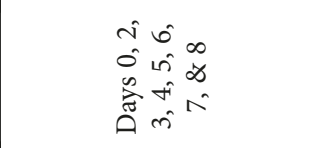 & $\underset{\sim}{\stackrel{\Im}{d}}$ & $\begin{array}{l}\hat{n} \\
\hat{n} \infty \\
\infty \\
\hat{\infty} \infty \\
\hat{\theta}\end{array}$ & 芒 & $\frac{\pi}{d}$ & $\stackrel{5}{\stackrel{5}{+\infty}}$ & $\begin{array}{l}\check{\Xi} \\
z\end{array}$ & 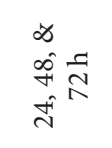 \\
\hline 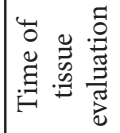 & 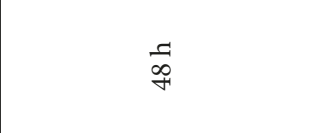 & $\underset{\sim}{\stackrel{f}{\sharp}}$ & 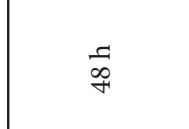 & 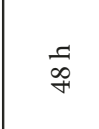 & $\underset{\sim}{\stackrel{I}{d}}$ & 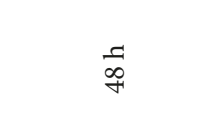 & $\underset{ }{\stackrel{J}{~}}$ & $\stackrel{\Xi}{N}$ \\
\hline 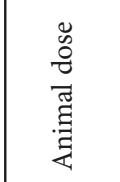 & 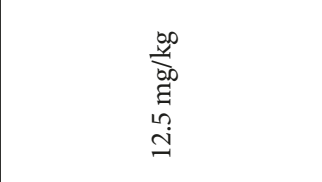 & 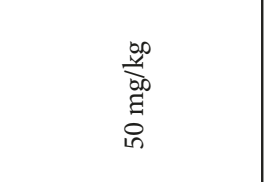 & 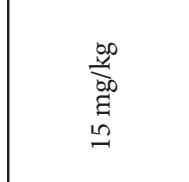 & 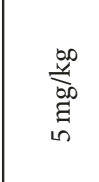 & 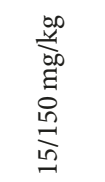 & 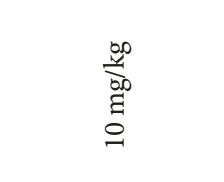 & 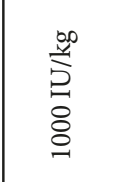 & 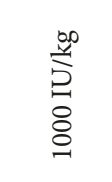 \\
\hline 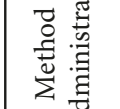 & ○ & $\Leftrightarrow$ & ᄋ & $\approx$ & $\Leftrightarrow$ & $\Leftrightarrow$ & $\approx$ & 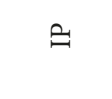 \\
\hline 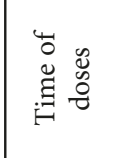 & 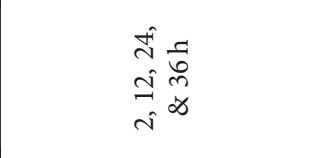 & $\begin{array}{l}\overrightarrow{1} \\
0 \\
\infty \\
\infty \\
0 \\
0\end{array}$ & 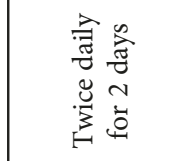 & 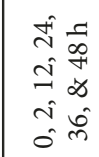 & $\stackrel{a}{\sim}$ & $\begin{array}{l}\infty \\
\text { d } \\
\text { di } \\
0 \\
0 \\
0\end{array}$ & 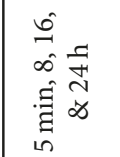 & 0 \\
\hline 䄈悹焉 & 芯 & $\begin{array}{l}\ddot{m} \\
\stackrel{0}{z}\end{array}$ & $\overrightarrow{\widetilde{\pi}}$ & 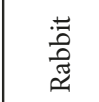 & 茞 & 茞 & 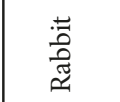 & $\begin{array}{l}\text { : } \\
\text { : } \\
\text { त्ञ }\end{array}$ \\
\hline $\begin{array}{l}\overrightarrow{\vec{z}} \\
\vec{D}\end{array}$ & 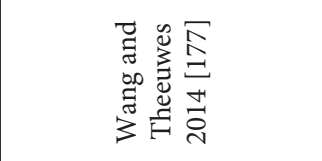 & 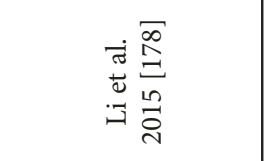 & 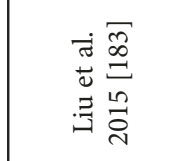 & 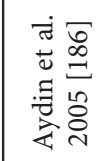 & 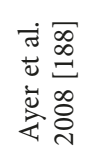 & 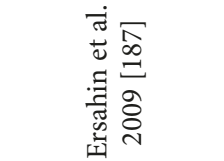 & 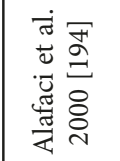 & 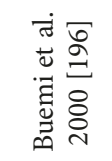 \\
\hline 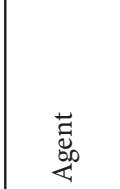 & 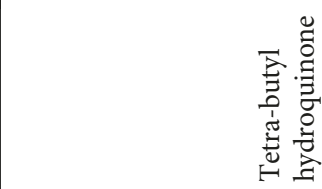 & & 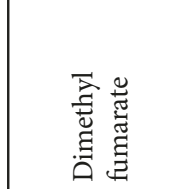 & & $\frac{5}{2}$ & & 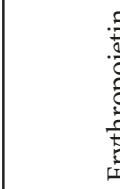 & 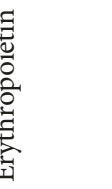 \\
\hline
\end{tabular}




\begin{tabular}{|c|c|c|c|c|c|c|c|}
\hline 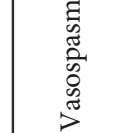 & 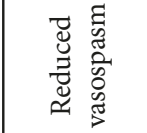 & 艺芯芯 & 艺薁 & 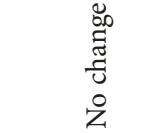 & 蒙总 & 艺蒡 & 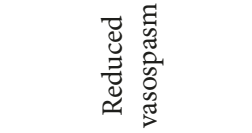 \\
\hline 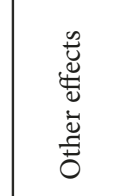 & 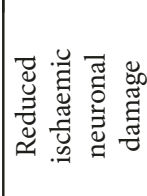 & 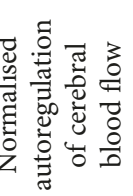 & 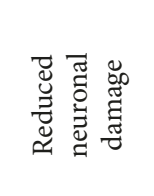 & 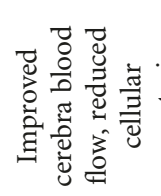 & 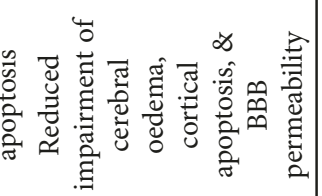 & 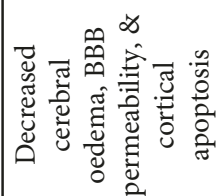 & 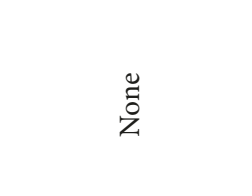 \\
\hline 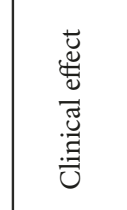 & 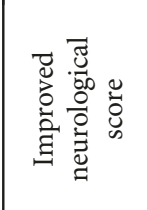 & 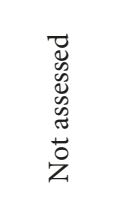 & 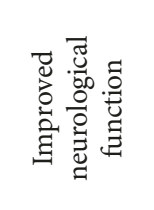 & 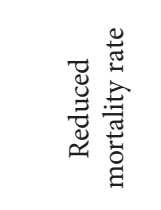 & 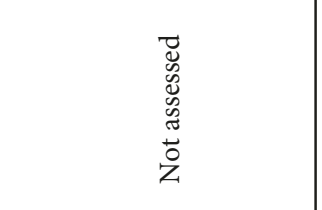 & 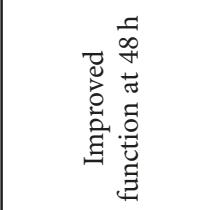 & 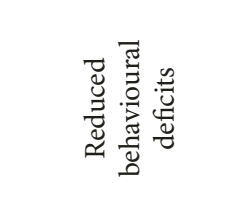 \\
\hline 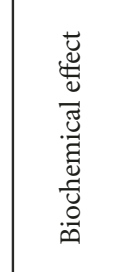 & 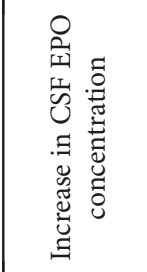 & 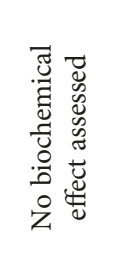 & 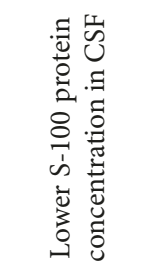 & 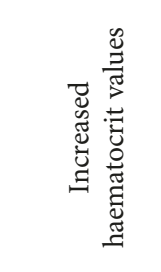 & 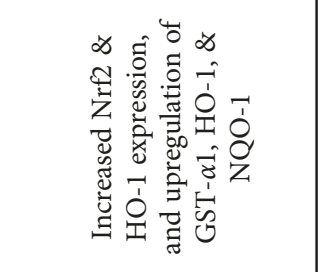 & 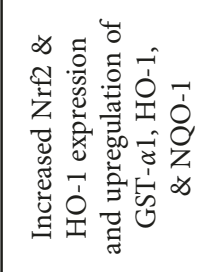 & 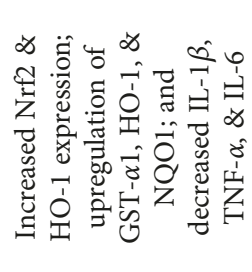 \\
\hline 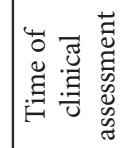 & $\underset{\mathbb{N}}{\stackrel{D}{N}}$ & 气̃̆ & $\frac{\pi}{N}$ & 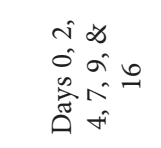 & 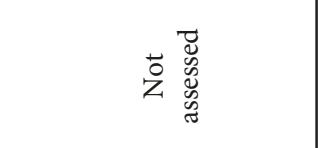 & 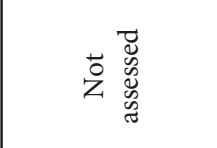 & $\underset{N}{\mathbb{N}}$ \\
\hline 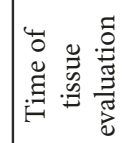 & $\stackrel{\mathbb{N}}{\mathbb{N}}$ & 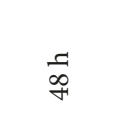 & $\underset{\mathbb{N}}{\vec{N}}$ & $\underset{\sim}{\stackrel{乛}{\sim}}$ & 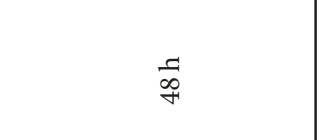 & 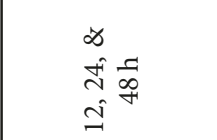 & $\stackrel{\mathbb{N}}{\mathbb{N}}$ \\
\hline 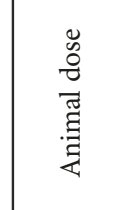 & 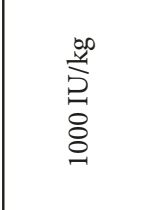 & 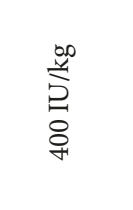 & 常 & 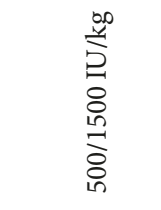 & 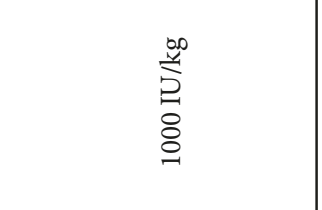 & 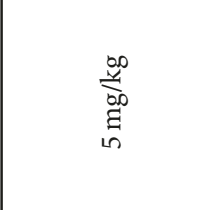 & 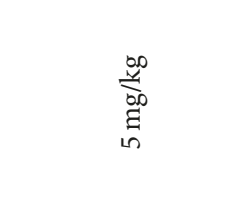 \\
\hline 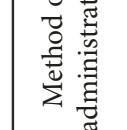 & $a$ & U & $a$ & $z$ & $\Leftrightarrow$ & $\Leftrightarrow$ & $\Leftrightarrow$ \\
\hline 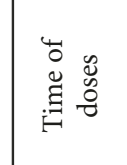 & 音 & 0 & 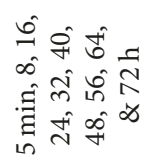 & $\begin{array}{l}\hat{+} \\
\hat{i} \\
\hat{0} \\
\hat{0} \\
\hat{0} \\
\hat{E}\end{array}$ & 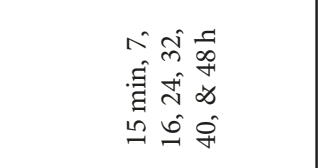 & 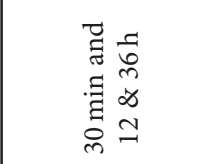 & 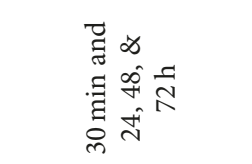 \\
\hline 尊悹离 & 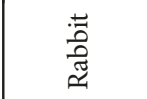 & 馬 & 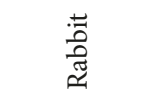 & 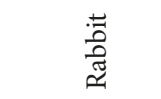 & 䲩 & $\stackrel{\overrightarrow{\widetilde{\Xi}}}{ }$ & 芯 \\
\hline 㺼 & 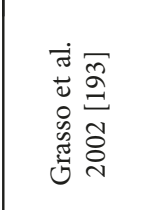 & 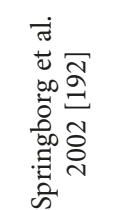 & 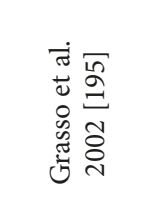 & 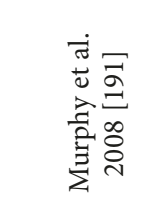 & 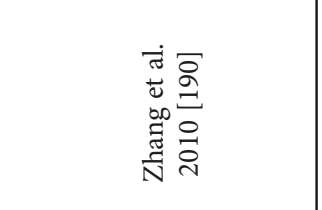 & 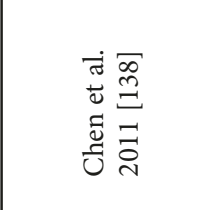 & 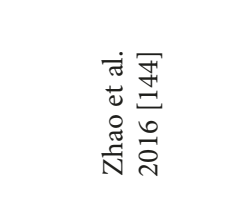 \\
\hline 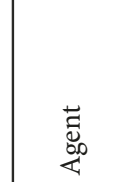 & & & & & & & 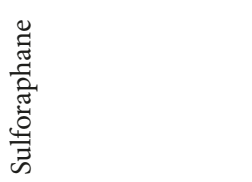 \\
\hline
\end{tabular}


As discussed, microvascular spasm may contribute more to poor outcome after SAH than macroscopic spasm of the main arteries. Microvascular spasm is more difficult to study experimentally, and the effect of SFN on this has not been reported, though unlikely to respond differently to SFN, compared to macrovascular spasm. Whichever predominates, both occur in a delayed manner three days to three weeks after SAH, which offers a good therapeutic window for treatment unlike most other types of ischaemic stroke.

There are no published clinical studies of SFN in humans after SAH. Indeed, there are no studies of direct SFN administration in humans at all, due to its relatively short half-life making clinical administration problematic. It has therefore been studied in the context of cruciferous vegetables. Cruciferous vegetables of the genus Brassica, including broccoli, cauliflower, Brussels sprouts, kale, collards, kohlrabi, and mustard, are a rich source of precursors of isothiocyanates called glucosinolates [153]. After ingestion of these vegetables, glucosinolates are hydrolysed by myrosinase; these two components, normally stored in separate subcellular locations, are brought together during digestion [154, 155]. Also, microorganisms in the colon have been shown to be involved in hydrolysis of glucosinolates into isothiocyanates [156]. Amongst all the cruciferous vegetables, broccoli in particular contains significant amounts of 4-methylsulfinylbutyl glucosinolate (4-MSB) or glucoraphanin [157] which can subsequently be converted to SFN.

The bioavailability of SFN has been studied in animals and humans. Due to its lipophilicity and molecular size, SFN is likely to passively diffuse through enterocytes. It is easily absorbed, conjugated to glutathione, and metabolised via the mercapturic acid pathway sequentially producing cysteinylglycine (SFN-CG), cysteine (SFN-Cys), and N-acetylcysteine (SFN-NAC) conjugates which are excreted in the urine [158]. SFN is primarily absorbed in the jejunum, and its bioavailability in humans is $74 \%$ [159]. In both humans and rats, approximately $70 \%$ of orally administered SFN is eliminated via the mercapturic acid pathway within 12-24 hours [160].

SFN has been demonstrated in the gastrointestinal and genitourinary tracts as well as the liver, pancreas, lung, and heart, albeit in varying concentrations; bioactivity may differ amongst organs [161]. In order for SFN to exert its neuroprotective effect, good CNS penetration is vital. One mouse study showed that SFN crossed the BBB and was detectable in the cerebral tissue including the midbrain and striatum between 15 and 120 minutes after intraperitoneal injection [162]. Another study demonstrated that at two and six hours after oral gavage, SFN metabolites were detectable in the CNS. Concentrations were relatively low, and SFN itself was no longer detectable, but this may be due to the relatively late time points selected [163]. Pragmatically, all previous experimental SAH studies have demonstrated potential benefits with peripherally administered SFN, and although peak concentration may be brief, there is evidence that repetitive stimulation with SFN can lead to elevated target mRNA for 24 hours and proteins for 48 hours [138, 144, 150]. Hence, with regular SFN dosing, a sustained upregulation of the protective Nrf2 transcriptome might be expected.
In many respects, SFN would appear to be an excellent candidate as a new therapeutic for patients after SAH. The lack of a practical formulation for clinical use has prevented trials to date. However, SFX-01 (Evgen Pharma) represents a novel solution to this by complexing SFN with $\alpha$-cyclodextrin to produce a stable powder that can be used clinically. A randomised controlled trial of SFX-01 after SAH is underway (NCT02614742).

5.1. Curcumin. Curcumin has been tested in several SAH models. In an in vitro SAH model where cortical neurons were exposed to $\mathrm{OxyHb}$, curcumin was shown to reduce oxidative stress, inflammatory cytokines including TNF- $\alpha$, IL-1 $\beta$, and IL-6, and neural apoptosis [164].

In a $\mathrm{SAH}$ perforation model, curcumin was administered at the time of injury and one, three, and 24 hours later. It reduced inflammatory cytokines and the rate of vasospasm and DCI. There was no associated improvement in neurological recovery using rotarod and open-field activity assessment up to day three, despite observing a maximum effect of haemorrhagic infarct volume at day six. Perhaps further behavioural testing should have been performed at a later stage to address this. Interestingly, only a single dose at the time of haemorrhage was found to be associated with reduction in cerebral infarction at day six as well as reduced MCA diameter three days after the haemorrhage. Other treatment time points were not associated with these observed benefits [165].

Similar improvements in the rate of vasospasm were seen in a recent study comparing the actions of nimodipine, nicorandil, and low and high-dose curcumin on cerebral vasospasm. This demonstrated that high-dose curcumin is associated with a lower rate of vasospasm compared to nimodipine and nicorandil [166]. Unfortunately, this study did not perform any behavioural testing. In addition, there is ambiguity regarding the exact timing of the treatment in relation to surgery. Although it is mentioned that only a single dose was given to all animals, further clarification regarding the timing of intervention as well as time points of the different analyses performed would be helpful before drawing any conclusions.

Animal behaviour was examined in a rat doublehaemorrhage model following intraperitoneal injection of curcumin three hours after SAH induction and daily thereafter for six days. Curcumin was shown to increase superoxide dismutase and catalase and reduce MDA levels in the cortex and hippocampus. Basilar artery perimeter and thickness were significantly altered in the treatment group indicating a reduction in vasospasm. There was reduced neuronal degeneration. Importantly, mortality was reduced and blinded neurological scores improved with curcumin. Curcumin-treated rats had a significantly lower mortality rate assessed during and after the induction of SAH compared to the other groups. Neurological scoring was performed at six hours and days one, three, five, and seven after the haemorrhage induction. Curcumin rats displayed better neurological scores up to day seven, but even in the untreated group, the neurological scores showed a positive trend on day seven [167]. This may suggest that the observed benefit may be lost if animals were to be assessed at a later 
time point. On the other hand, this finding could also represent the natural recovery from the disease.

Curcumin has been associated with an improvement in learning and memory impairment measured by the Morris water maze in a rat SAH model. Treatment duration with curcumin lasted for four weeks, and the authors claim that the positive benefit is secondary to downregulation of hippocampal TNF- $\alpha$ and inducible NOS. However, we were unable to make a full assessment of the manuscript as it is published in Chinese [168].

Curcumin has also been found to have benefits in ischaemic stroke similar to SFN. Nrf2 and HO-1 gene and protein levels were measured at three, six, 12, 24, 48, and 72 hours after MCA occlusion. An increase was seen at three hours, peaking at 24 hours poststroke. Infarct volume, brain water content, and early behavioural deficits assessed at 24 hours were reduced in the curcumin group [132].

5.2. Astaxanthin. Astaxantin (ASTX) is a carotenoid found in algae, fungi, complex plants, and seafood. It has been shown to be a powerful antioxidant [169]. The underlying mechanism of upregulation of Nrf2 by ASTX is not fully understood. However, it is thought that ASTX activates kinases such as phosphoinositol-3 kinase and extracellular signalregulated protein kinase which in turn upregulates the Nrf2 pathway [170]. In an experimental SAH model, ASTX was administered intrathecally 30 minutes after the induction of SAH. Animals were sacrificed at 24 hours, and tissues were evaluated. ASTX was shown to upregulate the expression of enzymes regulated by $\mathrm{Nrf} 2$ including $\mathrm{HO}-1$, NQO1, and GST- $\alpha 1$. Oxidative stress as measured by MDA levels was significantly reduced together with brain oedema, BBB disruption, and apoptosis. Neurological and behavioural deficits at 24 hours following SAH were improved [171]. These results were similar to their previous study which demonstrated the neuroprotective benefits of delayed treatment with oral ASTX, which started three hours post-SAH [172].

5.3. Lycopene. Lycopene is a natural carotenoid found mainly in tomatoes. It has multiple pleiotropic effects including antioxidant and anti-inflammatory actions [173] and neuroprotection from ischaemia [174]. At least some of its actions have been demonstrated to be due to upregulation of the Nrf2 pathway leading to neuroprotection in an experimental ischaemic model [175].

Lycopene has been tested in a rat SAH model. It was given once, two hours after SAH. Brain oedema, BBB disruption, and cortical apoptosis were significantly reduced at 24 hours. Neurology was only assessed at 24 hours, when neurological dysfunction was markedly reduced. The study showed a beneficial effect of lycopene due to reduction in inflammation as shown by downregulation of IL- $1 \beta$ and ICAM-1, but whether this was mediated through Nrf2 was not specifically investigated [176].

A phase II clinical trial assessing the effect of lycopene on cerebral vasospasm and autoregulation after SAH has been registered (NCT00905931). It has recruited 15 patients to date but is currently on hold due to temporary problems with IMP availability (personal communication).
5.4. Tetra-butyl Hydroquinone. Tetra-butyl hydroquinone (tBHQ) has been evaluated in two SAH models [177, 178]. In mice 24 hours after haemorrhage, there was no evidence of Nrf2 upregulation by tBHQ. However, at 48 hours after haemorrhage, tBHQ upregulated the expression of KEAP1, Nrf2, HO-1, NQO1, and GST $\alpha 1$ [178]. These animals displayed less brain oedema, BBB impairment, cortical apoptosis, and neurodegeneration. The treatment was started at two hours and repeated at 12, 24, and 36 hours after SAH. The study included two groups. The first group was decapitated at 48 hours, and tissues were evaluated. This showed marked upregulation of Nrf2 in neurons and glial cells. In the second experiment, the rats were trained and evaluated in a Morris water maze demonstrating significant improvement in performance and learning deficits following tBHQ on days four and five. Further memory testing up to day 8 showed no significant difference following the use of $\mathrm{tBHQ}$ [177].

5.5. Dimethyl Fumarate. Dimethyl fumarate (DMF) is an ester of fumaric acid conventionally used in the treatment of psoriasis [179]. DMF has been shown to modulate inflammation in the brain and specifically in multiple sclerosis (MS) through activation of the KEAP1-Nrf2-ARE pathway $[180,181]$. Following a randomised phase III clinical trial showing that DMF ameliorated relapsing-remitting MS [182], it has been repurposed and is being used clinically for this indication.

The effects of DMF have been investigated in a rat prechiasmatic cistern injection model utilising autologous blood in rats. DMF was administered orally twice daily for two days, but the exact timing of administration relative to SAH was not specified. Two sets of experiments were performed. In the first group, tissue analysis took place only once 48 hours after surgery. The second experiment involved Morris water maze assessment of trained animals up to five days after the haemorrhage. Activities of KEAP1, Nrf2, and HO-1 were significantly increased within glial cells and neurons of animals treated with DMF. Cortical MDA was decreased, and superoxide dismutase and glutathione peroxidase activities were increased. Levels of proinflammatory cytokines IL- $1 \beta$, TNF- $\alpha$, and IL- 6 were reduced. Behavioural assessments in the group treated with DMF showed marked improvement in the performance of the treatment group which was more evident on days four and five [183].

5.6. Melatonin. Melatonin is a well-known antioxidant with the ability to scavenge free radicals probably acting through multiple mechanisms $[184,185]$. Studies have shown attenuation of early brain injury and vasospasm [186] with improvement in early neurological function (assessed at 48 hours) with a once daily regimen [187] and reduction in mortality rate (assessed within 24 hours of SAH induction) [188]. Furthermore, the mechanism behind these actions was linked to activation of Nrf2 [189].

In a rat SAH model, animals were treated with intraperitoneal injection of melatonin $150 \mathrm{mg} / \mathrm{kg}$ at two and 24 hours after the induction of SAH. Neurological scores and brain tissues were examined at 48 hours. Nrf2 and HO- 1 were upregulated at 48 hours in the SAH group, mainly expressed 
on neurons. The levels of HO-1, NQO1, and GST- $\alpha 1$ mRNA were significantly increased in the cortex following treatment with melatonin. Brain oedema, BBB dysfunction, and cortical apoptosis were all reduced. Within the time frame of the experiment, early assessments of behavioural deficits of animals were significantly reduced in the treatment arm [189].

5.7. Erythropoietin. Erythropoietin (EPO) is a pleiotropic molecule with known effects on Nrf2. In an experimental SAH model, EPO was injected intraperitoneally five minutes after SAH and every eight hours up to 48 hours. HO-1, NQO1, and GST $-\alpha 1$ were all upregulated. Cortical apoptosis, brain oedema, and BBB impairment were all significantly reduced in the EPO-treated group. Although EPO is a pleiotropic molecule, the upregulation of Nrf2 proteins supports the mechanism of action through activation of the Nrf2ARE pathway, hence reducing oxidative stress [190]. Other than its effects on early brain injury, further experimental studies in SAH made strong suggestions of potential benefits of EPO including improved cerebral blood flow [191, 192] autoregulation [192], reduction in vasoconstriction [193], post-SAH cerebral ischaemia [193, 194], and early improvement in behavioural function [193, 195, 196], and one study even claimed a reduction in mortality rate although this was only measured up to 72 hours [196].

EPO is unique amongst the agents identified in having been assessed in human studies. In a small case series of seven patients, EPO was shown to be effective in improving brain tissue oxygen tension if given over three consecutive days. This showed anti-inflammatory properties as well as restoration of cerebral autoregulation [197]. So far, two doubleblinded placebo-controlled randomised trials [198, 199] have failed to demonstrate benefit with high-dose intravenous EPO. However, these studies were small (73 and 80 patients) and not adequately powered to show a clinical difference. Tseng et al. did observe a trend towards a reduced incidence of severe vasospasm, and a review concluded that EPO possibly reduces the severity of the cerebral vasospasm but not its incidence [200]. These trials have however demonstrated the safety of EPO following SAH and allayed any safety concerns over EPO and its association with increased risk of thrombosis [201]. The latter is important since SAH is a condition where patients are already in a hypercoagulable state and historically haemodilution has been advocated. However, further larger clinical trials would be required to address the efficacy of EPO.

5.8. Summary. There is good experimental evidence suggesting that early Nrf2 activation reduces deficits early after SAH although more studies examining their effects on long-term outcome are needed. The reasons underlying the paucity of studies examining long-term functional outcome are unclear. This may be due to poor experimental design, practical reasons, or difficulty in inducing significant late deficits without excessive early mortality in rodent SAH models. Other than EPO, there have been no completed human clinical trials of Nrf2 activation in SAH. Experimental studies suggest biochemical and early functional improvements following treatment, although it is difficult to test for the more subtle neurocognitive deficits most prevalent in patients with SAH. The timing of administration of first dose in animal studies was generally early (often within 2 hours of SAH), with few studies providing data on later use. Although this is a potential concern for human studies, even if data on later administration was available in animal models, extrapolation of the therapeutic window from animals to humans is notoriously difficult if not impossible, and given the generally much slower evolution of SAH in humans compared to rodents, trials administering at the earliest available opportunity, up to 72 hours after ictus when patients start to deteriorate, could be considered. There are a number of potential agents that could be used in this context. There are no head-to-head comparisons in the literature, and they are all reported to penetrate the CNS, have relatively good safety profiles, and with exception of EPO, can be given orally. There is therefore little to guide which may be most suitable.

\section{Conclusion}

Outcomes following SAH remain poor despite advances in treatment. The mechanisms underlying recovery from SAH are multifactorial; however, Nrf2 activation appears to play a key protective role. There is overwhelming evidence for the therapeutic potential of several Nrf2 activators, with studies replicated in different SAH models and different laboratories. In the absence of any human data, there is a clear need for clinical studies to examine the safety and efficacy of Nrf2 activation after SAH.

\section{Abbreviations}

ARE: Antioxidant response element

BACH1: BTB and CNC homology 1

CBP: $\quad$ CREB-binding protein

HO-1: Heme-oxygenase 1

HP: Haptoglobin

KEAP1: Kelch-like ECH-associated protein 1

MAF: Musculoaponeurotic fibrosarcoma

Nrf2: $\quad$ Nuclear factor-erythroid 2- (NF-E2-) related factor 2

P: $\quad$ Phosphate group

PG: $\quad$ Prostaglandin

S(R): $\quad$ Sulphide side chain reduced by a group $\mathrm{R}$

SH: $\quad$ Sulfhydryl side chain.

\section{Conflicts of Interest}

Diederik Bulters is the chief investigator, and Ian Galea and Ardalan Zolnourian are investigators of the SAS (SFX-01 after SAH) trial sponsored by Evgen Pharma.

\section{Authors' Contributions}

Ian Galea and Diederik Bulters are joint senior authors.

\section{Acknowledgments}

This work has not been funded by an external body. Ardalan Zolnourian's research fellowship has been granted by Royal 
College of Surgeons of England. We thank Freya Davis (BSc Hons, Pharmacology), who has kindly proofread the manuscript.

\section{References}

[1] R. Sercombe, Y. R. T. Dinh, and P. Gomis, "Cerebrovascular inflammation following subarachnoid hemorrhage," The Japanese Journal of Pharmacology, vol. 88, no. 3, pp. 227249, 2002.

[2] T. Al-Khindi, R. Macdonald, and T. Schweizer, "Cognitive and functional outcome after aneurysmal subarachnoid hemorrhage," Stroke, vol. 41, no. 8, pp. e519-e536, 2010.

[3] M. R. Quigley and M. Salary, "Defining survivorship after high-grade aneurysmal subarachnoid hemorrhage," Surgical Neurology, vol. 69, no. 3, pp. 261-265, 2008.

[4] A. Pace, S. Mitchell, E. Casselden et al., "A subarachnoid haemorrhage-specific outcome tool," Brain, vol. 141, no. 4, pp. 1111-1121, 2018.

[5] K. T. Kreiter, D. Copeland, G. L. Bernardini et al., "Predictors of cognitive dysfunction after subarachnoid hemorrhage," Stroke, vol. 33, no. 1, pp. 200-209, 2002.

[6] R. M. Pluta, "Delayed cerebral vasospasm and nitric oxide: review, new hypothesis, and proposed treatment," Pharmacology \& Therapeutics, vol. 105, no. 1, pp. 23-56, 2005.

[7] P. Gaetani, A. Pasqualin, R. Rodriguez y Baena, E. Borasio, and F. Marzatico, "Oxidative stress in the human brain after subarachnoid hemorrhage," Journal of Neurosurgery, vol. 89, no. 5, pp. 748-754, 1998.

[8] K. Fassbender, K. Fassbender, B. Hodapp et al., "Inflammatory cytokines in subarachnoid haemorrhage: association with abnormal blood flow velocities in basal cerebral arteries," Journal of Neurology, Neurosurgery \& Psychiatry, vol. 70, no. 4, pp. 534-537, 2001.

[9] J. P. Dreier, S. Major, A. Manning et al., "Cortical spreading ischaemia is a novel process involved in ischaemic damage in patients with aneurysmal subarachnoid haemorrhage," Brain, vol. 132, no. 7, pp. 1866-1881, 2009.

[10] F. A. Pennings, G. J. Bouma, and C. Ince, "Direct observation of the human cerebral microcirculation during aneurysm surgery reveals increased arteriolar contractility," Stroke, vol. 35, no. 6, pp. 1284-1288, 2004.

[11] N. W. C. Dorsch and M. T. King, "A review of cerebral vasospasm in aneurysmal subarachnoid haemorrhage part I: incidence and effects," Journal of Clinical Neuroscience, vol. 1, no. 1, pp. 19-26, 1994.

[12] H. Nornes, "The role of intracranial pressure in the arrest of hemorrhage in patients with ruptured intracranial aneurysm," Journal of Neurosurgery, vol. 39, no. 2, pp. 226-234, 1973.

[13] B. Voldby and E. M. Enevoldsen, "Intracranial pressure changes following aneurysm rupture. Part 3: recurrent hemorrhage," Journal of Neurosurgery, vol. 56, no. 6, pp. 784789, 1982.

[14] T. Fukuhara, C. M. Douville, J. P. Eliott, D. W. Newell, and H. R. Winn, "Relationship between intracranial pressure and the development of vasospasm after aneurysmal subarachnoid hemorrhage," Neurologia Medico-Chirurgica, vol. 38, no. 11, pp. 710-717, 1998.

[15] J. M. Schmidt, S. B. Ko, R. Helbok et al., "Cerebral perfusion pressure thresholds for brain tissue hypoxia and metabolic crisis after poor-grade subarachnoid hemorrhage," Stroke, vol. 42, no. 5, pp. 1351-1356, 2011.

[16] G. G. Heuer, M. J. Smith, J. P. Elliott, H. R. Winn, and P. D. Leroux, "Relationship between intracranial pressure and other clinical variables in patients with aneurysmal subarachnoid hemorrhage," Journal of Neurosurgery, vol. 101, no. 3, pp. 408-416, 2004.

[17] M. Jaeger, M. U. Schuhmann, M. Soehle, C. Nagel, and J. Meixensberger, "Continuous monitoring of cerebrovascular autoregulation after subarachnoid hemorrhage by brain tissue oxygen pressure reactivity and its relation to delayed cerebral infarction," Stroke, vol. 38, no. 3, pp. 981-986, 2007.

[18] K. P. Budohoski, M. Czosnyka, P. Smielewski et al., "Impairment of cerebral autoregulation predicts delayed cerebral ischemia after subarachnoid hemorrhage: a prospective observational study," Stroke, vol. 43, no. 12, pp. 32303237, 2012.

[19] T. Dóczi, "The pathogenetic and prognostic significance of blood-brain barrier damage at the acute stage of aneurysmal subarachnoid haemorrhage. Clinical and experimental studies," Acta Neurochirurgica, vol. 77, no. 3-4, pp. 110132, 1985.

[20] F. A. László, C. Varga, and T. Dóczi, "Cerebral oedema after subarachnoid haemorrhage. Pathogenetic significance of vasopressin," Acta Neurochirurgica, vol. 133, no. 3-4, pp. 122-133, 1995.

[21] M. Zetterling, L. Hallberg, and E. Ronne-Engström, "Early global brain oedema in relation to clinical admission parameters and outcome in patients with aneurysmal subarachnoid haemorrhage," Acta Neurochirurgica, vol. 152, no. 9, pp. 1527-1533, 2010.

[22] W. J. Cahill, J. H. Calvert, and J. H. Zhang, "Mechanisms of early brain injury after subarachnoid hemorrhage," Journal of Cerebral Blood Flow and Metabolism, vol. 26, no. 11, pp. 1341-1353, 2006.

[23] S. P. Lad, H. Hegen, G. Gupta, F. Deisenhammer, and G. K. Steinberg, "Proteomic biomarker discovery in cerebrospinal fluid for cerebral vasospasm following subarachnoid hemorrhage," Journal of Stroke and Cerebrovascular Diseases, vol. 21, no. 1, pp. 30-41, 2012.

[24] G. Kusaka, M. Ishikawa, A. Nanda, D. N. Granger, and J. H. Zhang, "Signaling pathways for early brain injury after subarachnoid hemorrhage," Journal of Cerebral Blood Flow \& Metabolism, vol. 24, no. 8, pp. 916-925, 2004.

[25] M. J. McGirt, M. Bydon, K. R. Archer et al., "Serum von Willebrand factor, matrix metalloproteinase-9, and vascular endothelial growth factor levels predict the onset of cerebral vasospasm after aneurysmal subarachnoid hemorrhage," Neurosurgery, vol. 51, no. 5, pp. 1128-1135, 2002.

[26] M. Siuta, S. L. Zuckerman, and J. Mocco, "Nitric oxide in cerebral vasospasm: theories, measurement, and treatment," Neurology Research International, vol. 2013, Article ID 972417, 11 pages, 2013.

[27] R. M. Pluta, B. G. Thompson, T. M. Dawson, S. H. Snyder, R. J. Boock, and E. H. Oldfield, "Loss of nitric oxide synthase immunoreactivity in cerebral vasospasm," Journal of Neurosurgery, vol. 84, no. 4, pp. 648-654, 1996.

[28] M. D. I. Vergouwen, M. Vermeulen, J. van Gijn et al., "Definition of delayed cerebral ischemia after aneurysmal subarachnoid hemorrhage as an outcome event in clinical trials and observational studies: proposal of a 
multidisciplinary research group," Stroke, vol. 41, no. 10, pp. 2391-2395, 2010.

[29] N. Toda, K. Shimizu, and T. Ohta, "Mechanism of cerebral arterial contraction induced by blood constituents," Journal of Neurosurgery, vol. 53, no. 3, pp. 312-322, 1980.

[30] N. Toda, "Mechanisms of contracting action of oxyhemoglobin in isolated monkey and dog cerebral arteries," American Journal of Physiology-Heart and Circulatory Physiology, vol. 258, no. 1, pp. H57-H63, 1990.

[31] R. L. Macdonald and B. K. Weir, "A review of hemoglobin and the pathogenesis of cerebral vasospasm," Stroke, vol. 22, no. 8, pp. 971-982, 1991.

[32] S. Nishizawa and I. Laher, "Signaling mechanisms in cerebral vasospasm," Trends in Cardiovascular Medicine, vol. 15, no. 1, pp. 24-34, 2005.

[33] R. P. Jewell, C. M. Saundry, A. D. Bonev, B. I. Tranmer, and G. C. Wellman, "Inhibition of Ca++ sparks by oxyhemoglobin in rabbit cerebral arteries," Journal of Neurosurgery, vol. 100, no. 2, pp. 295-302, 2004.

[34] R. P. Ostrowski, A. R. Colohan, and J. H. Zhang, "Molecular mechanisms of early brain injury after subarachnoid hemorrhage," Neurological Research, vol. 28, no. 4, pp. 399414, 2006

[35] T. Mori, K. Nagata, T. Town, J. Tan, T. Matsui, and T. Asano, "Intracisternal increase of superoxide anion production in a canine subarachnoid hemorrhage model," Stroke, vol. 32, no. 3, pp. 636-642, 2001.

[36] P. G. Matz, M. Fujimura, and P. H. Chan, "Subarachnoid hemolysate produces DNA fragmentation in a pattern similar to apoptosis in mouse brain," Brain Research, vol. 858, no. 2, pp. 312-319, 2000.

[37] P. G. Matz, J.-C. Copin, P. H. Chan, and R. L. Macdonald, "Cell death after exposure to subarachnoid hemolysate correlates inversely with expression of CuZn-superoxide dismutase," Stroke, vol. 31, no. 10, pp. 2450-2459, 2000.

[38] Y.-W. Cheng, W.-J. Li, X.-J. Dou et al., "Role of endothelin-1 and its receptors in cerebral vasospasm following subarachnoid hemorrhage," Molecular Medicine Reports, vol. 18, pp. 5229-5236, 2018.

[39] V. Seifert, B. M. Löffler, M. Zimmermann, S. Roux, and D. Stolke, "Endothelin concentrations in patients with aneurysmal subarachnoid hemorrhage: correlation with cerebral vasospasm, delayed ischemic neurological deficits, and volume of hematoma," Journal of Neurosurgery, vol. 82, pp. 55-62, 1995.

[40] M. Ishiguro, T. L. Wellman, A. Honda, S. R. Russell, B. I. Tranmer, and G. C. Wellman, "Emergence of a R-type Ca2 + channel (Cav 2.3) contributes to cerebral artery constriction after subarachnoid hemorrhage," Circulation Research, vol. 96, no. 4, pp. 419-426, 2005.

[41] A. Can, R. F. Rudy, V. M. Castro et al., "Low serum calcium and magnesium levels and rupture of intracranial aneurysms," Stroke, vol. 49, no. 7, pp. 1747-1750, 2018.

[42] Y. R. Tran Dinh, A. Jomaa, J. Callebert et al., "Overexpression of cyclooxygenase-2 in rabbit basilar artery endothelial cells after subarachnoid hemorrhage," Neurosurgery, vol. 48, no. 3, pp. 626-635, 2001.

[43] M. Nosko, R. Schulz, B. Weir, D. A. Cook, and M. Grace, "Effects of vasospasm on levels of prostacyclin and thromboxane A2 in cerebral arteries of the monkey," Neurosurgery, vol. 22, no. 1, pp. 45-50, 1988.
[44] J. P. Dreier, J. Woitzik, M. Fabricius et al., "Delayed ischaemic neurological deficits after subarachnoid haemorrhage are associated with clusters of spreading depolarizations," Brain, vol. 129, no. 12, pp. 3224-3237, 2006.

[45] A. G. Harders and J. M. Gilsbach, "Time course of blood velocity changes related to vasospasm in the circle of Willis measured by transcranial Doppler ultrasound," Journal of Neurosurgery, vol. 66, pp. 718-728, 1987.

[46] C. M. Fisher, J. P. Kistler, and J. M. Davis, "Relation of cerebral vasospasm to subarachnoid hemorrhage visualized by computerized tomographic scanning," Neurosurgery, vol. 6, no. 1, pp. 1-9, 1980.

[47] J. Claassen, G. L. Bernardini, K. Kreiter et al., "Effect of cisternal and ventricular blood on risk of delayed cerebral ischemia after subarachnoid hemorrhage: The fisher scale revisited," Stroke, vol. 32, no. 9, pp. 2012-2020, 2001.

[48] G. Kumar, R. B. Shahripour, and M. R. Harrigan, "Vasospasm on transcranial Doppler is predictive of delayed cerebral ischemia in aneurysmal subarachnoid hemorrhage: a systematic review and meta-analysis," Journal of Neurosurgery, vol. 124, no. 5, pp. 1257-1264, 2016.

[49] A. A. Rabinstein, J. A. Friedman, S. D. Weigand et al., "Predictors of cerebral infarction in aneurysmal subarachnoid hemorrhage," Stroke, vol. 35, no. 8, pp. 1862-1866, 2004.

[50] R. L. Macdonald, R. T. Higashida, E. Keller et al., "Randomised trial of clazosentan, an endothelin receptor antagonist, in patients with aneurysmal subarachnoid hemorrhage undergoing surgical clipping (CONSCIOUS2)," in Cerebral Vasospasm: Neurovascular Events after Subarachnoid Hemorrhage, M. Zuccarello, J. Clark, G. PyneGeithman, N. Andaluz, J. Hartings, and O. Adeoye, Eds., vol. 115 of Acta Neurochirurgica Supplement, pp. 27-31, Springer, Vienna, 2013.

[51] M. Naraoka, N. Matsuda, N. Shimamura, K. Asano, and H. Ohkuma, "The role of arterioles and the microcirculation in the development of vasospasm after aneurysmal SAH," BioMed Research International, vol. 2014, Article ID 253746, 9 pages, 2014.

[52] D. A. Herz, S. Baez, and K. Shulman, "Pial microcirculation in subarachnoid hemorrhage," Stroke, vol. 6, no. 4, pp. 417424, 1975.

[53] H. Ohkuma, K. Itoh, S. Shibata, and S. Suzuki, "Morphological changes of intraparenchymal arterioles after experimental subarachnoid hemorrhage in dogs," Neurosurgery, vol. 41, no. 1, pp. 230-236, 1997.

[54] M. Sabri, J. Ai, K. Lakovic, J. D’abbondanza, D. Ilodigwe, and R. L. Macdonald, "Mechanisms of microthrombi formation after experimental subarachnoid hemorrhage," Neuroscience, vol. 224, pp. 26-37, 2012.

[55] B. Friedrich, F. Müller, S. Feiler, K. Schöller, and N. Plesnila, "Experimental subarachnoid hemorrhage causes early and long-lasting microarterial constriction and microthrombosis: an in-vivo microscopy study," Journal of Cerebral Blood Flow and Metabolism, vol. 32, no. 3, pp. 447-455, 2012.

[56] H. Ohkuma, H. Manabe, M. Tanaka, and S. Suzuki, "Impact of cerebral microcirculatory changes on cerebral blood flow during cerebral vasospasm after aneurysmal subarachnoid hemorrhage," Stroke, vol. 31, no. 7, pp. 1621-1627, 2000.

[57] G. Neil-Dwyer, D. A. Lang, B. Doshi, C. J. Gerber, and P. W. Smith, "Delayed cerebral ischaemia: the pathological substrate," Acta Neurochirurgica, vol. 131, no. 1-2, pp. 137$145,1994$. 
[58] R. J. Brown, A. Kumar, R. Dhar, T. R. Sampson, and M. N. Diringer, "The relationship between delayed infarcts and angiographic vasospasm after aneurysmal subarachnoid hemorrhage," Neurosurgery, vol. 72, no. 5, pp. 702-708, 2013.

[59] S. Weidauer, H. Lanfermann, A. Raabe, F. Zanella, V. Seifert, and J. Beck, "Impairment of cerebral perfusion and infarct patterns attributable to vasospasm after aneurysmal subarachnoid hemorrhage: a prospective MRI and DSA study," Stroke, vol. 38, no. 6, pp. 1831-1836, 2007.

[60] K. R. Wagner, F. R. Sharp, T. D. Ardizzone, A. Lu, and J. F. Clark, "Heme and iron metabolism: role in cerebral hemorrhage," Journal of Cerebral Blood Flow \& Metabolism, vol. 23, no. 6, pp. 629-652, 2003.

[61] G. Balla, G. M. Vercellotti, U. Muller-Eberhard, J. Eaton, and H. S. Jacob, "Exposure of endothelial cells to free heme potentiates damage mediated by granulocytes and toxic oxygen species," Laboratory Investigation, vol. 64 , no. 5, pp. 648655, 1991.

[62] T. Kamezaki, K. Yanaka, S. Nagase, K. Fujita, N. Kato, and T. Nose, "Increased levels of lipid peroxides as predictive of symptomatic vasospasm and poor outcome after aneurysmal subarachnoid hemorrhage," Journal of Neurosurgery, vol. 97, no. 6, pp. 1302-1305, 2002.

[63] Y. P. Hsieh, C. L. Lin, A. L. Shiue et al., "Correlation of F4-neuroprostanes levels in cerebrospinal fluid with outcome of aneurysmal subarachnoid hemorrhage in humans," Free Radical Biology \& Medicine, vol. 47, no. 6, pp. 814824, 2009.

[64] S. Zhang, L. Wang, M. Liu, and B. Wu, "Tirilazad for aneurysmal subarachnoid haemorrhage," Cochrane Database of Systematic Reviews, no. 2, article CD006778, 2010.

[65] J. F. Clark and F. R. Sharp, "Bilirubin oxidation products (BOXes) and their role in cerebral vasospasm after subarachnoid hemorrhage," Journal of Cerebral Blood Flow and Metabolism, vol. 26, no. 10, pp. 1223-1233, 2006.

[66] G. J. Pyne-Geithman, C. J. Morgan, K. Wagner et al., "Bilirubin production and oxidation in CSF of patients with cerebral vasospasm after subarachnoid hemorrhage," Journal of Cerebral Blood Flow and Metabolism, vol. 25, no. 8, pp. 1070-1077, 2005.

[67] I. Laher and J. H. Zhang, "Protein kinase C and cerebral vasospasm," Journal of Cerebral Blood Flow \& Metabolism, vol. 21, no. 8, pp. 887-906, 2001.

[68] K. Yamashita, Y. Kotani, Y. Nakajima et al., "Fasudil, a Rho kinase (ROCK) inhibitor, protects against ischemic neuronal damage in vitro and in vivo by acting directly on neurons," Brain Research, vol. 1154, pp. 215-224, 2007.

[69] A. Saito, M. Inoue, H. Kon et al., "Effectiveness of intraarterial administration of fasudil hydrochloride for preventing symptomatic vasospasm after subarachnoid hemorrhage," in Neurovascular Events after Subarachnoid Hemorrhage, J. Fandino, S. Marbacher, A. R. Fathi, C. Muroi, and E. Keller, Eds., vol. 120 of Acta Neurochirurgica Supplement, pp. 297-301, Springer, Cham, 2015.

[70] G. L. Gallia and R. J. Tamargo, "Leukocyte-endothelial cell interactions in chronic vasospasm after subarachnoid hemorrhage," Neurological Research, vol. 28, no. 7, pp. 750758, 2006.

[71] D. Graetz, A. Nagel, F. Schlenk, O. Sakowitz, P. Vajkoczy, and A. Sarrafzadeh, "High ICP as trigger of proinflammatory IL-6 cytokine activation in aneurysmal subarachnoid hemorrhage," Neurological Research, vol. 32, no. 7, pp. 728-735, 2010.
[72] T. Mathiesen, B. Andersson, A. Loftenius, and H. von Holst, "Increased interleukin-6 levels in cerebrospinal fluid following subarachnoid hemorrhage," Journal of Neurosurgery, vol. 78, no. 4, pp. 562-567, 1993.

[73] T. Mathiesen, G. Edner, E. Ulfarsson, and B. Andersson, "Cerebrospinal fluid interleukin-1 receptor antagonist and tumor necrosis factor-alpha following subarachnoid hemorrhage," Journal of Neurosurgery, vol. 87, no. 2, pp. 215220, 1997.

[74] K. A. Hanafy, B. Grobelny, L. Fernandez et al., "Brain interstitial fluid TNF- $\alpha$ after subarachnoid hemorrhage," Journal of the Neurological Sciences, vol. 291, no. 1-2, pp. 69-73, 2010.

[75] A. S. Dumont, R. J. Dumont, M. M. Chow et al., "Cerebral vasospasm after subarachnoid hemorrhage: putative role of inflammation," Neurosurgery, vol. 53, no. 1, pp. 123-135, 2003.

[76] J. Galea, K. Ogungbenro, S. Hulme et al., "Reduction of inflammation after administration of interleukin-1 receptor antagonist following aneurysmal subarachnoid hemorrhage: results of the Subcutaneous Interleukin-1Ra in SAH (SCILSAH) study," Journal of Neurosurgery, vol. 128, no. 2, pp. 515-523, 2017.

[77] V. Banwell, E. S. Sena, and M. R. Macleod, "Systematic review and stratified meta-analysis of the efficacy of interleukin-1 receptor antagonist in animal models of stroke," Journal of Stroke and Cerebrovascular Diseases, vol. 18, no. 4, pp. 269276, 2009.

[78] A. D. Greenhalgh, D. Brough, E. M. Robinson, S. Girard, N. J. Rothwell, and S. M. Allan, "Interleukin-1 receptor antagonist is beneficial after subarachnoid haemorrhage in rat by blocking haem-driven inflammatory pathology," Disease Models \& Mechanisms, vol. 5, no. 6, pp. 823-833, 2012.

[79] C. Holmes, C. Cunningham, E. Zotova et al., "Systemic inflammation and disease progression in alzheimer disease," Neurology, vol. 73, no. 10, pp. 768-774, 2009.

[80] R. J. Guerreiro, I. Santana, J. M. Brás, B. Santiago, A. Paiva, and C. Oliveira, "Peripheral inflammatory cytokines as biomarkers in Alzheimer's disease and mild cognitive impairment," Neurodegenerative Diseases, vol. 4, no. 6, pp. 406-412, 2007.

[81] J. P. Dreier and C. Reiffurth, "The stroke-migraine depolarization continuum," Neuron, vol. 86, no. 4, pp. 902-922, 2015.

[82] M. Lauritzen, J. P. Dreier, M. Fabricius, J. A. Hartings, R. Graf, and A. J. Strong, "Clinical relevance of cortical spreading depression in neurological disorders: migraine, malignant stroke, subarachnoid and intracranial hemorrhage, and traumatic brain injury," Journal of Cerebral Blood Flow \& Metabolism, vol. 31, no. 1, pp. 17-35, 2011.

[83] O. Windmüller, U. Lindauer, M. Foddis et al., "Ion changes in spreading ischaemia induce rat middle cerebral artery constriction in the absence of NO," Brain, vol. 128, no. 9, pp. 2042-2051, 2005.

[84] O. R. Hubschmann and D. Kornhauser, "Cortical cellular response in acute subarachnoid hemorrhage," Journal of Neurosurgery, vol. 52, no. 4, pp. 456-462, 1980.

[85] J. P. Dreier, K. Körner, N. Ebert et al., "Nitric oxide scavenging by hemoglobin or nitric oxide synthase inhibition by $\mathrm{N}$-nitro$\mathrm{L}$-arginine induces cortical spreading ischemia when $\mathrm{K}^{+}$is increased in the subarachnoid space," Journal of Cerebral Blood Flow \& Metabolism, vol. 18, no. 9, pp. 978-990, 1998.

[86] J. P. Dreier, N. Ebert, J. Priller et al., "Products of hemolysis in the subarachnoid space inducing spreading ischemia in the 
cortex and focal necrosis in rats: a model for delayed ischemic neurological deficits after subarachnoid hemorrhage?," Journal of Neurosurgery, vol. 93, no. 4, pp. 658-666, 2000.

[87] G. C. Petzold, S. Haack, O. von Bohlen und Halbach et al., "Nitric oxide modulates spreading depolarization threshold in the human and rodent cortex," Stroke, vol. 39, no. 4, pp. 1292-1299, 2008.

[88] G. C. Petzold, K. M. Einhäupl, U. Dirnagl, and J. P. Dreier, "Ischemia triggered by spreading neuronal activation is induced by endothelin-1 and hemoglobin in the subarachnoid space," Annals of Neurology, vol. 54, no. 5, pp. 591-598, 2003.

[89] J. P. Dreier, "The role of spreading depression, spreading depolarization and spreading ischemia in neurological disease," Nature Medicine, vol. 17, no. 4, pp. 439-447, 2011.

[90] J. P. Dreier, M. Fabricius, C. Ayata et al., "Recording, analysis, and interpretation of spreading depolarizations in neurointensive care: review and recommendations of the COSBID research group," Journal of Cerebral Blood Flow and Metabolism, vol. 37, no. 5, pp. 1595-1625, 2017.

[91] D. von Bornstädt, T. Houben, J. L. Seidel et al., "Supplydemand mismatch transients in susceptible peri-infarct hot zones explain the origins of spreading injury depolarizations," Neuron, vol. 85, no. 5, pp. 1117-1131, 2015.

[92] O. Hubschmann and D. Kornhauser, "Effect of subarachnoid hemorrhage on the extracellular microenvironment," Journal of Neurosurgery, vol. 56, no. 2, pp. 216-221, 1982.

[93] C. Beaulieu, E. Busch, A. De Crespigny, and M. E. Moseley, "Spreading waves of transient and prolonged decreases in water diffusion after subarachnoid hemorrhage in rats," Magnetic Resonance in Medicine, vol. 44, no. 1, pp. 110116, 2000.

[94] J. Woitzik, J. P. Dreier, N. Hecht et al., "Delayed cerebral ischemia and spreading depolarization in absence of angiographic vasospasm after subarachnoid hemorrhage," Journal of Cerebral Blood Flow \& Metabolism, vol. 32, no. 2, pp. 203212, 2012.

[95] A. Urbach, C. Bruehl, and O. W. Witte, "Microarray-based long-term detection of genes differentially expressed after cortical spreading depression," The European Journal of Neuroscience, vol. 24, no. 3, pp. 841-856, 2006.

[96] K. Itoh, T. Chiba, S. Takahashi et al., "An Nrf2/small Maf heterodimer mediates the induction of phase II detoxifying enzyme genes through antioxidant response elements," Biochemical and Biophysical Research Communications, vol. 236, no. 2, pp. 313-322, 1997.

[97] R. Venugopal and A. K. Jaiswal, "Nrf2 and Nrf1 in association with Jun proteins regulate antioxidant response elementmediated expression and coordinated induction of genes encoding detoxifying enzymes," Oncogene, vol. 17, no. 24, pp. 3145-3156, 1998.

[98] X. Zhao, G. Sun, S. M. Ting et al., "Cleaning up after ICH: The role of Nrf2 in modulating microglia function and hematoma clearance," Journal of Neurochemistry, vol. 133, no. 1, pp. 144-152, 2015.

[99] X. Zhao, S. Song, G. Sun et al., "Neuroprotective role of haptoglobin after intracerebral hemorrhage," The Journal of Neuroscience, vol. 29, no. 50, pp. 15819-15827, 2009.

[100] C. M. Morris, J. M. Candy, J. A. Edwardson, C. A. Bloxham, and A. Smith, "Evidence for the localization of haemopexin immunoreactivity in neurones in the human brain," Neuroscience Letters, vol. 149, no. 2, pp. 141-144, 1993.
[101] M. Chen and R. F. Regan, "Time course of increased heme oxygenase activity and expression after experimental intracerebral hemorrhage: correlation with oxidative injury," Journal of Neurochemistry, vol. 103, no. 5, pp. 2015-2021, 2007.

[102] N. Harada, M. Kanayama, A. Maruyama et al., "Nrf2 regulates ferroportin 1-mediated iron efflux and counteracts lipopolysaccharide-induced ferroportin 1 mRNA suppression in macrophages," Archives of Biochemistry and Biophysics, vol. 508, no. 1, pp. 101-109, 2011.

[103] C. A. ALPER, J. H. PETERS, A. G. BIRTCH, and F. H. GARDNER, "Haptoglobin synthesis. I. in vivo studies of the production of haptoglobin, fibrinogen, and $\gamma$-globulin by the canine liver," The Journal of Clinical Investigation, vol. 44, no. 4, pp. 574-581, 1965.

[104] J. Greer, W. D. L. Liao, and W. E. Brown, "Haptoglobinhemoglobin complex. Subunit interaction probed by crosslinking," Journal of Biological Chemistry, vol. 256, pp. 87718774, 1981.

[105] M.-Y. Lee, S. Y. Kim, J. S. Choi et al., "Upregulation of haptoglobin in reactive astrocytes after transient forebrain ischemia in rats," Journal of Cerebral Blood Flow \& Metabolism, vol. 22, no. 10, pp. 1176-1180, 2002.

[106] M. Kristiansen, J. H. Graversen, C. Jacobsen et al., "Identification of the haemoglobin scavenger receptor," Nature, vol. 409, no. 6817, pp. 198-201, 2001.

[107] J. Galea, G. Cruickshank, J. L. Teeling et al., "The intrathecal CD163-haptoglobin-hemoglobin scavenging system in subarachnoid hemorrhage," Journal of Neurochemistry, vol. 121, no. 5, pp. 785-792, 2012.

[108] M. R. Langlois and J. R. Delanghe, "Biological and clinical significance of haptoglobin polymorphism in humans," Clinical Chemistry, vol. 42, no. 10, pp. 1589-1600, 1996.

[109] M. Borsody, A. Burke, W. Coplin, R. Miller-Lotan, and A. Levy, "Haptoglobin and the development of cerebral artery vasospasm after subarachnoid hemorrhage," Neurology, vol. 66, no. 5, pp. 634-640, 2006.

[110] J. L. Leclerc, S. Blackburn, D. Neal et al., "Haptoglobin phenotype predicts the development of focal and global cerebral vasospasm and may influence outcomes after aneurysmal subarachnoid hemorrhage," Proceedings of the National Academy of Sciences, vol. 112, no. 4, pp. 11551160, 2015.

[111] S. B. Murthy, J. Caplan, A. P. Levy et al., "Haptoglobin 2-2 genotype is associated with cerebral salt wasting syndrome in aneurysmal subarachnoid hemorrhage," Neurosurgery, vol. 78, no. 1, pp. 71-76, 2015.

[112] B. Gaastra, J. Glazier, D. Bulters, and I. Galea, "Haptoglobin genotype and outcome after subarachnoid haemorrhage: new insights from a meta-analysis," Oxidative Medicine and Cellular Longevity, vol. 2017, Article ID 6747940, 9 pages, 2017.

[113] S. B. Murthy, A. P. Levy, J. Duckworth et al., "Presence of haptoglobin-2 allele is associated with worse functional outcomes after spontaneous intracerebral hemorrhage," World Neurosurgery, vol. 83, no. 4, pp. 583-587, 2015.

[114] E. Kantor, H. Bayır, D. Ren et al., "Haptoglobin genotype and functional outcome after aneurysmal subarachnoid hemorrhage," Journal of Neurosurgery, vol. 120, no. 2, pp. 386390, 2014.

[115] J. Gburek, P. J. Verroust, T. E. Willnow et al., "Megalin and cubilin are endocytic receptors involved in renal clearance 
of hemoglobin," Journal of the American Society of Nephrology, vol. 13, no. 2, pp. 423-430, 2002.

[116] V. Hvidberg, M. B. Maniecki, C. Jacobsen, P. Højrup, H. J. Møller, and S. K. Moestrup, "Identification of the receptor scavenging hemopexin-heme complexes," Blood, vol. 106, no. 7, pp. 2572-2579, 2005.

[117] A. T. Dinkova-Kostova, W. D. Holtzclaw, and T. W. Kensler, "The role of Keap1 in cellular protective responses," Chemical Research in Toxicology, vol. 18, no. 12, pp. 1779-1791, 2005.

[118] D. A. Bloom and A. K. Jaiswal, "Phosphorylation of Nrf2 at $\mathrm{Ser}^{40}$ by protein kinase $\mathrm{C}$ in response to antioxidants leads to the release of Nrf2 from INrf2, but is not required for Nrf2 stabilization/accumulation in the nucleus and transcriptional activation of antioxidant response element," Journal of Biological Chemistry, vol. 278, no. 45, pp. 44675-44682, 2003.

[119] S. B. Cullinan, D. Zhang, M. Hannink, E. Arvisais, R. J. Kaufman, and J. A. Diehl, "Nrf2 is a direct PERK substrate and effector of PERK-dependent cell survival," Molecular and Cellular Biology, vol. 23, no. 20, pp. 7198-7209, 2003.

[120] H. C. Huang, T. Nguyen, and C. B. Pickett, "Phosphorylation of Nrf2 at Ser- 40 by protein kinase $\mathrm{C}$ regulates antioxidant response element-mediated transcription," The Journal of Biological Chemistry, vol. 277, no. 45, pp. 42769-42774, 2002.

[121] K. Itoh, T. Ishii, N. Wakabayashi, and M. Yamamoto, "Regulatory mechanisms of cellular response to oxidative stress," Free Radical Research, vol. 31, no. 4, pp. 319-324, 1999.

[122] T. Johansen and T. Lamark, "Selective autophagy mediated by autophagic adapter proteins," Autophagy, vol. 7, no. 3, pp. 279-296, 2011.

[123] A. Jain, T. Lamark, E. Sjøttem et al., "p62/SQSTM1 is a target gene for transcription factor NRF2 and creates a positive feedback loop by inducing antioxidant response elementdriven gene transcription," Journal of Biological Chemistry, vol. 285, no. 29, pp. 22576-22591, 2010.

[124] A. Lau, X. J. Wang, F. Zhao et al., "A noncanonical mechanism of Nrf2 activation by autophagy deficiency: direct interaction between Keap1 and p62," Molecular and Cellular Biology, vol. 30, no. 13, pp. 3275-3285, 2010.

[125] M. Komatsu, H. Kurokawa, S. Waguri et al., "The selective autophagy substrate p62 activates the stress responsive transcription factor Nrf2 through inactivation of Keap1," Nature Cell Biology, vol. 12, no. 3, pp. 213-223, 2010.

[126] G. H. Liu, J. Qu, and X. Shen, "NF- $\kappa$ B/p65 antagonizes Nrf2ARE pathway by depriving CBP from Nrf2 and facilitating recruitment of HDAC3 to MafK," Biochimica et Biophysica Acta (BBA) - Molecular Cell Research, vol. 1783, no. 5, pp. 713-727, 2008.

[127] S. Dhakshinamoorthy, A. K. Jain, D. A. Bloom, and A. K. Jaiswal, "Bach1 competes with Nrf2 leading to negative regulation of the antioxidant response element (ARE)-mediated $\mathrm{NAD}(\mathrm{P}) \mathrm{H}$ :quinone oxidoreductase 1 gene expression and induction in response to antioxidants," The Journal of Biological Chemistry, vol. 280, no. 17, pp. 16891-16900, 2005.

[128] M. Sandberg, J. Patil, B. D'Angelo, S. G. Weber, and C. Mallard, "NRF2-regulation in brain health and disease: implication of cerebral inflammation," Neuropharmacology, vol. 79, pp. 298-306, 2014.

[129] N. Tanaka, Y. Ikeda, Y. Ohta et al., "Expression of Keap1Nrf2 system and antioxidative proteins in mouse brain after transient middle cerebral artery occlusion," Brain Research, vol. 1370, pp. 246-253, 2011.
[130] J. Dang, L. O. Brandenburg, C. Rosen et al., "Nrf2 expression by neurons, astroglia, and microglia in the cerebral cortical penumbra of ischemic rats," Journal of Molecular Neuroscience, vol. 46, no. 3, pp. 578-584, 2012.

[131] S. Srivastava, A. Alfieri, R. C. M. Siow, G. E. Mann, and P. A. Fraser, "Temporal and spatial distribution of Nrf2 in rat brain following stroke: quantification of nuclear to cytoplasmic Nrf2 content using a novel immunohistochemical technique," The Journal of Physiology, vol. 591, no. 14, pp. 3525-3538, 2013.

[132] C. Yang, X. Zhang, H. Fan, and Y. Liu, "Curcumin upregulates transcription factor $\mathrm{Nrf2}$, HO-1 expression and protects rat brains against focal ischemia," Brain Research, vol. 1282, pp. 133-141, 2009.

[133] A. Y. Shih, “A small-molecule-inducible Nrf2-mediated antioxidant response provides effective prophylaxis against cerebral ischemia in vivo," The Journal of Neuroscience, vol. 25, no. 44, pp. 10321-10335, 2005.

[134] Z. A. Shah, R. C. Li, R. K. Thimmulappa et al., "Role of reactive oxygen species in modulation of Nrf2 following ischemic reperfusion injury," Neuroscience, vol. 147, no. 1, pp. 53-59, 2007.

[135] J. Wang, J. Fields, C. Zhao et al., "Role of Nrf2 in protection against intracerebral hemorrhage injury in mice," Free Radical Biology \& Medicine, vol. 43, no. 3, pp. 408-414, 2007.

[136] Y. Yang, Z. Xi, Y. Xue et al., "Hemoglobin pretreatment endows rat cortical astrocytes resistance to hemininduced toxicity via Nrf2/HO-1 pathway," Experimental Cell Research, vol. 361, no. 2, pp. 217-224, 2017.

[137] Z. Wang, G. Chen, W. W. Zhu, and D. Zhou, "Activation of nuclear factor-erythroid 2-related factor 2 (Nrf2) in the basilar artery after subarachnoid hemorrhage in rats," Annals of Clinical and Laboratory Science, vol. 40, no. 3, pp. 233239, 2010

[138] G. Chen, Q. Fang, J. Zhang, D. Zhou, and Z. Wang, "Role of the Nrf2-ARE pathway in early brain injury after experimental subarachnoid hemorrhage," Journal of Neuroscience Research, vol. 89, no. 4, pp. 515-523, 2011.

[139] H. Pan, H. Wang, L. Zhu, L. Mao, L. Qiao, and X. Su, "Depletion of $\mathrm{Nrf} 2$ enhances inflammation induced by oxyhemoglobin in cultured mice astrocytes," Neurochemical Research, vol. 36, no. 12, pp. 2434-2441, 2011.

[140] T. Li, H. Wang, Y. Ding et al., "Genetic elimination of Nrf2 aggravates secondary complications except for vasospasm after experimental subarachnoid hemorrhage in mice," Brain Research, vol. 1558, pp. 90-99, 2014.

[141] K. Kowalczuk and M. Stryjecka-Zimmer, "The influence of oxidative stress on the level of malondialdehyde (MDA) in different areas of the rabbit brain," Annales Universitatis Mariae Curie-Sklodowska. Sectio D: Medicina, vol. 57, no. 2, pp. 160-164, 2002.

[142] D. A. Butterfield, C. B. Pocernich, and J. Drake, "Elevated glutathione as a therapeutic strategy in Alzheimer's disease," Drug Development Research, vol. 56, no. 3, pp. 428-437, 2002.

[143] M. Kemp, Y.-M. Go, and D. P. Jones, "Nonequilibrium thermodynamics of thiol/disulfide redox systems: a perspective on redox systems biology," Free Radical Biology \& Medicine, vol. 44, no. 6, pp. 921-937, 2008.

[144] X. Zhao, L. Wen, M. Dong, and X. Lu, "Sulforaphane activates the cerebral vascular Nrf2-ARE pathway and suppresses inflammation to attenuate cerebral vasospasm in rat 
with subarachnoid hemorrhage," Brain Research, vol. 1653, pp. 1-7, 2016.

[145] G. Wells, "Peptide and small molecule inhibitors of the Keap1-Nrf2 protein-protein interaction," Biochemical Society Transactions, vol. 43, no. 4, pp. 674-679, 2015.

[146] B. N. R. Jaja, G. Saposnik, H. F. Lingsma et al., "Development and validation of outcome prediction models for aneurysmal subarachnoid haemorrhage: The SAHIT multinational cohort study," BMJ, vol. 360, article j5745, 2018.

[147] A. Durnford, J. Dunbar, J. Galea et al., "Haemoglobin scavenging after subarachnoid haemorrhage," in Neurovascular Events after Subarachnoid Hemorrhage, J. Fandino, S. Marbacher, A. R. Fathi, C. Muroi, and E. Keller, Eds., vol. 120 of Acta Neurochirurgica Supplement, pp. 51-54, Springer, Cham, 2015.

[148] Y. Li, J. D. Paonessa, and Y. Zhang, "Mechanism of chemical activation of Nrf2," PLoS One, vol. 7, no. 4, article e35122, 2012.

[149] R. K. Thimmulappa, K. H. Mai, and S. Srisuma, "Identification of Nrf2-regulated genes induced by the chemopreventive agent sulforaphane by oligonucleotide microarray," vol. 62, no. 18, pp. 5196-5203, 2002.

[150] X. D. Zhao, Y. T. Zhou, and X. J. Lu, "Sulforaphane enhances the activity of the Nrf2-ARE pathway and attenuates inflammation in OxyHb-induced rat vascular smooth muscle cells," Inflammation Research, vol. 62, no. 9, pp. 857-863, 2013.

[151] A. Alfieri, S. Srivastava, R. C. M. Siow et al., "Sulforaphane preconditioning of the Nrf2/HO-1 defense pathway protects the cerebral vasculature against blood-brain barrier disruption and neurological deficits in stroke," Free Radical Biology \& Medicine, vol. 65, pp. 1012-1022, 2013.

[152] J. Zhao, N. Kobori, J. Aronowski, and P. K. Dash, "Sulforaphane reduces infarct volume following focal cerebral ischemia in rodents," Neuroscience Letters, vol. 393, no. 2-3, pp. 108-112, 2006.

[153] A. R. Kristal and J. W. Lampe, "Brassica vegetables and prostate cancer risk: a review of the epidemiological evidence," Nutrition and Cancer, vol. 42, no. 1, pp. 1-9, 2002.

[154] T. A. Shapiro, J. W. Fahey, K. L. Wade, K. K. Stephenson, and P. Talalay, "Human metabolism and excretion of cancer chemoprotective glucosinolates and isothiocyanates of cruciferous vegetables," Cancer Epidemiology, Biomarkers \& Prevention, vol. 7, pp. 1091-1100, 1998.

[155] T. A. Shapiro, J. W. Fahey, K. L. Wade, K. K. Stephenson, and P. Talalay, "Chemoprotective glucosinolates and isothiocyanates of broccoli sprouts: metabolism and excretion in humans," Cancer Epidemiology, Biomarkers \& Prevention, vol. 10, pp. 501-508, 2001.

[156] F. J. Barba, N. Nikmaram, S. Roohinejad, A. Khelfa, Z. Zhu, and M. Koubaa, "Bioavailability of glucosinolates and their breakdown products: impact of processing," Frontiers in Nutrition, vol. 3, p. 24, 2016.

[157] I. Schonhof, A. Krumbein, and B. Brückner, "Genotypic effects on glucosinolates and sensory properties of broccoli and cauliflower," Nahrung - Food, vol. 48, no. 1, pp. 2533, 2004

[158] P. A. Egner, T. W. Kensler, J. G. Chen, S. J. Gange, J. D. Groopman, and M. D. Friesen, "Quantification of sulforaphane mercapturic acid pathway conjugates in human urine by high-performance liquid chromatography and isotope- dilution tandem mass spectrometry," Chemical Research in Toxicology, vol. 21, no. 10, pp. 1991-1996, 2008.

[159] N. Petri, C. Tannergren, B. Holst et al., "Absorption/ metabolism of sulforaphane and quercetin, and regulation of phase ii enzymes, in human jejunum in vivo," Drug Metabolism and Disposition, vol. 31, no. 6, pp. 805-813, 2003.

[160] P. A. Egner, J. G. Chen, J. B. Wang et al., "Bioavailability of sulforaphane from two broccoli sprout beverages: results of a short-term, cross-over clinical trial in Qidong, China," Cancer Prevention Research, vol. 4, no. 3, pp. 384-395, 2011.

[161] A. Tarozzi, C. Angeloni, M. Malaguti, F. Morroni, S. Hrelia, and P. Hrelia, "Sulforaphane as a potential protective phytochemical against neurodegenerative diseases," Oxidative Medicine and Cellular Longevity, vol. 2013, Article ID 415078, 10 pages, 2013.

[162] A. Jazwa, A. I. Rojo, N. G. Innamorato, M. Hesse, J. Fernández-Ruiz, and A. Cuadrado, "Pharmacological targeting of the transcription factor Nrf2 at the basal ganglia provides disease modifying therapy for experimental parkinsonism," Antioxidants \& Redox Signaling, vol. 14, no. 12, pp. 2347-2360, 2011.

[163] J. D. Clarke, A. Hsu, D. E. Williams et al., "Metabolism and tissue distribution of sulforaphane in Nrf2 knockout and wild-type mice," Pharmaceutical Research, vol. 28, no. 12, pp. 3171-3179, 2011.

[164] X. Li, L. Zhao, L. Yue et al., "Evidence for the protective effects of curcumin against oxyhemoglobin-induced injury in rat cortical neurons," Brain Research Bulletin, vol. 120, pp. 3440, 2015.

[165] C. Wakade, M. D. King, M. D. Laird, C. H. Alleyne Jr., and K. M. Dhandapani, "Curcumin attenuates vascular inflammation and cerebral vasospasm after subarachnoid hemorrhage in mice," Antioxidants \& Redox Signaling, vol. 11, no. 1, pp. 35-46, 2009.

[166] H. E. Aydin, N. E. Bektur, Z. Ozbek, S. Oner, C. Baycu, and F. S. Kilic, "Comparison of the effects and mechanism of the curcumin with different drugs in experimental vasospasm after subarachnoid hemorrhage," Turkish Neurosurgery, vol. 27, pp. 884-893, 2017.

[167] C.-P. Kuo, C. H. Lu, L. L. Wen et al., "Neuroprotective effect of curcumin in an experimental rat model of subarachnoid hemorrhage," Anesthesiology, vol. 115, no. 6, pp. 12291238, 2011.

[168] Z. Qiu and S. Yue, "Curcumin improves learning and memory function through decreasing hippocampal TNF- $\alpha$ and iNOS levels after subarachnoid hemorrhage in rats," Xi Bao Yu Fen Zi Mian Yi Xue Za Zhi, vol. 32, pp. 343346, 2016.

[169] D. N. Tripathi and G. B. Jena, “Astaxanthin intervention ameliorates cyclophosphamide-induced oxidative stress, DNA damage and early hepatocarcinogenesis in rat: role of Nrf2, p53, p38 and phase-II enzymes," Mutation Research/Genetic Toxicology and Environmental Mutagenesis, vol. 696, no. 1, pp. 69-80, 2010.

[170] J. Kim, S. Nam, B. Kim, and W. Choi, “Astaxanthin improves stem cell potency via an increase in the proliferation of neural progenitor cells," International Journal of Molecular Sciences, vol. 11, no. 12, pp. 5109-5119, 2010.

[171] Q. Wu, X. S. Zhang, H. D. Wang et al., "Astaxanthin activates nuclear factor erythroid-related factor 2 and the antioxidant responsive element (nrf2-are) pathway in the brain after 
subarachnoid hemorrhage in rats and attenuates early brain injury," Marine Drugs, vol. 12, no. 12, pp. 6125-6141, 2014.

[172] X.-S. Zhang, X. Zhang, M. L. Zhou et al., "Amelioration of oxidative stress and protection against early brain injury by astaxanthin after experimental subarachnoid hemorrhage," Journal of Neurosurgery, vol. 121, no. 1, pp. 42-54, 2014.

[173] B. C. Ip, K. Q. Hu, C. Liu et al., "Lycopene metabolite, apo$10^{\prime}$-lycopenoic acid, inhibits diethylnitrosamine-initiated, high fat diet-promoted hepatic inflammation and tumorigenesis in mice," Cancer Prevention Research, vol. 6, no. 12, pp. 1304-1316, 2013.

[174] G. Hsiao, T. H. Fong, N. H. Tzu, K. H. Lin, D. S. Chou, and J. R. Sheu, "A potent antioxidant, lycopene, affords neuroprotection against microglia activation and focal cerebral ischemia in rats," In Vivo, vol. 18, no. 3, pp. 351-356, 2004.

[175] X. Lei, L. Lei, Z. Zhang, and Y. Cheng, "Neuroprotective effects of lycopene pretreatment on transient global cerebral ischemia-reperfusion in rats: the role of the $\mathrm{Nrf} 2 / \mathrm{HO}-1$ signaling pathway," Molecular Medicine Reports, vol. 13, no. 1, pp. 412-418, 2016.

[176] A. Wu, R. Liu, W. Dai et al., "Lycopene attenuates early brain injury and inflammation following subarachnoid hemorrhage in rats," International Journal of Clinical and Experimental Medicine, vol. 8, no. 8, pp. 1431614322, 2015.

[177] Z. Wang and J. Theeuwes, "Tert-butylhydroquinone alleviates early brain injury and cognitive dysfunction after experimental subarachnoid hemorrhage: role of Keap1/Nrf2/ARE pathway," PLoS One, vol. 9, no. 5, article e97685, 2014.

[178] T. Li, K. J. Sun, H. D. Wang et al., “Tert-butylhydroquinone ameliorates early brain injury after experimental subarachnoid hemorrhage in mice by enhancing Nrf2-independent autophagy," Neurochemical Research, vol. 40, no. 9, pp. 1829-1838, 2015.

[179] R. Gold, R. A. Linker, and M. Stangel, "Fumaric acid and its esters: an emerging treatment for multiple sclerosis with antioxidative mechanism of action," Clinical Immunology, vol. 142, no. 1, pp. 44-48, 2012.

[180] H. Wilms, J. Sievers, U. Rickert, M. Rostami-Yazdi, U. Mrowietz, and R. Lucius, "Dimethylfumarate inhibits microglial and astrocytic inflammation by suppressing the synthesis of nitric oxide, IL- $1 \beta$, TNF- $\alpha$ and IL- 6 in an in-vitro model of brain inflammation," Journal of Neuroinflammation, vol. 7, no. 1, pp. 30-38, 2010.

[181] R. A. Linker, D. H. Lee, S. Ryan et al., "Fumaric acid esters exert neuroprotective effects in neuroinflammation via activation of the Nrf2 antioxidant pathway," Brain, vol. 134, no. 3, pp. 678-692, 2011.

[182] R. Gold, L. Kappos, D. L. Arnold et al., "Placebo-controlled phase 3 study of oral BG-12 for relapsing multiple sclerosis," The New England Journal of Medicine, vol. 367, no. 12, pp. 1098-1107, 2012.

[183] Y. Liu, J. Qiu, Z. Wang et al., "Dimethylfumarate alleviates early brain injury and secondary cognitive deficits after experimental subarachnoid hemorrhage via activation of Keap1-Nrf2-ARE system," Journal of Neurosurgery, vol. 123, no. 4, pp. 915-923, 2015.

[184] L. Mao, H. D. Wang, X. L. Wang, L. Tian, and J. Y. Xu, "Disruption of Nrf2 exacerbated the damage after spinal cord injury in mice," The Journal of Trauma and Acute Care Surgery, vol. 72, pp. 1-198, 2012.
[185] A. Galano, D. X. Tan, and R. J. Reiter, "Melatonin as a natural ally against oxidative stress: a physicochemical examination," Journal of Pineal Research, vol. 51, no. 1, pp. 1-16, 2011.

[186] M. V. Aydin, H. Caner, O. Sen et al., "Effect of melatonin on cerebral vasospasm following experimental subarachnoid hemorrhage," Neurological Research, vol. 27, no. 1, pp. 7782, 2005.

[187] M. Ersahin, H. Z. Toklu, Ş. Çetinel, M. Yüksel, B. Ç. Yeğen, and G. Şener, "Melatonin reduces experimental subarachnoid hemorrhage-induced oxidative brain damage and neurological symptoms," Journal of Pineal Research, vol. 46, no. 3, pp. 324-332, 2009.

[188] R. E. Ayer, T. Sugawara, W. Chen, W. Tong, and J. H. Zhang, "Melatonin decreases mortality following severe subarachnoid hemorrhage," Journal of Pineal Research, vol. 44, no. 2, pp. 197-204, 2008.

[189] Z. Wang, C. Ma, C. J. Meng et al., "Melatonin activates the Nrf2-ARE pathway when it protects against early brain injury in a subarachnoid hemorrhage model," Journal of Pineal Research, vol. 53, no. 2, pp. 129-137, 2012.

[190] J. Zhang, Y. Zhu, D. Zhou, Z. Wang, and G. Chen, "Recombinant human erythropoietin (rhEPO) alleviates early brain injury following subarachnoid hemorrhage in rats: possible involvement of Nrf2-ARE pathway," Cytokine, vol. 52, no. 3, pp. 252-257, 2010.

[191] A. M. Murphy, A. Xenocostas, P. Pakkiri, and T.-Y. Lee, "Hemodynamic effects of recombinant human erythropoietin on the central nervous system after subarachnoid hemorrhage: reduction of microcirculatory impairment and functional deficits in a rabbit model," Journal of Neurosurgery, vol. 109, no. 6, pp. 1155-1164, 2008.

[192] J. B. Springborg, X. D. Ma, P. Rochat et al., “A single subcutaneous bolus of erythropoietin normalizes cerebral blood flow autoregulation after subarachnoid haemorrhage in rats," pp. 823-829, 2002.

[193] G. Grasso, M. Buemi, C. Alafaci et al., "Beneficial effects of systemic administration of recombinant human erythropoietin in rabbits subjected to subarachnoid hemorrhage," Proceedings of the National Academy of Sciences, vol. 99, no. 8, pp. 5627-5631, 2002.

[194] C. Alafaci, F. Salpietro, G. Grasso et al., "Effect of recombinant human erythropoietin on cerebral ischemia following experimental subarachnoid hemorrhage," European Journal of Pharmacology, vol. 406, no. 2, pp. 219-225, 2000.

[195] G. Grasso, M. Passalacqua, A. Sfacteria et al., "Does administration of recombinant human erythropoietin attenuate the increase of S-100 protein observed in cerebrospinal fluid after experimental subarachnoid hemorrhage?," Journal of Neurosurgery, vol. 96, pp. 565-570, 2002.

[196] M. Buemi, G. Grasso, F. Corica et al., "In vivo evidence that erythropoietin has a neuroprotective effect during subarachnoid hemorrhage," European Journal of Pharmacology, vol. 392, no. 1-2, pp. 31-34, 2000.

[197] R. Helbok, E. Shaker, R. Beer et al., "High dose erythropoietin increases brain tissue oxygen tension in severe vasospasm after subarachnoid hemorrhage," BMC Neurology, vol. 12, no. $1,2012$.

[198] J. B. Springborg, C. Møller, P. Gideon, O. S. Jørgensen, M. Juhler, and N. V. Olsen, "Erythropoietin in patients with aneurysmal subarachnoid haemorrhage: a double blind randomised clinical trial," Acta Neurochirurgica, vol. 149, no. 11, pp. 1089-1101, 2007. 
[199] M.-Y. Tseng, P. J. Hutchinson, H. K. Richards et al., “Acute systemic erythropoietin therapy to reduce delayed ischemic deficits following aneurysmal subarachnoid hemorrhage: a phase II randomized, double-blind, placebo-controlled trial," Journal of Neurosurgery, vol. 111, no. 1, pp. 171180, 2009.

[200] J. D. Turner, A. Mammis, and C. J. Prestigiacomo, "Erythropoietin for the treatment of subarachnoid hemorrhage: a review," World Neurosurgery, vol. 73, no. 5, pp. 500-507, 2010.

[201] W. Jelkmann, "Physiology and pharmacology of erythropoietin," Transfusion Medicine and Hemotherapy, vol. 40, no. 5, pp. 302-309, 2013.

[202] R. Jäger, R. P. Lowery, A. V. Calvanese, J. M. Joy, M. Purpura, and J. M. Wilson, "Comparative absorption of curcumin formulations," Nutrition Journal, vol. 13, no. 1, p. 11, 2014.

[203] J. M. Odeberg, Å. Lignell, A. Pettersson, and P. Höglund, "Oral bioavailability of the antioxidant astaxanthin in humans is enhanced by incorporation of lipid based formulations," European Journal of Pharmaceutical Sciences, vol. 19, no. 4, pp. 299-304, 2003.

[204] D. M. Gustin, K. A. Rodvold, J. A. Sosman et al., "Singledose pharmacokinetic study of lycopene delivered in a well-defined food-based lycopene delivery system (tomato paste-oil mixture) in healthy adult male subjects," Cancer Epidemiology, Biomarkers \& Prevention, vol. 13, pp. 850860, 2004.

[205] J. Sun, H. Hu, X. Ren, and J. W. Simpkins, "Tert-butylhydroquinone compromises survival in murine experimental stroke," Neurotoxicology and Teratology, vol. 54, pp. 1521, 2016 .

[206] Z. Al-Jaderi and A. A. Maghazachi, "Utilization of dimethyl fumarate and related molecules for treatment of multiple sclerosis, cancer, and other diseases," Frontiers in Immunology, vol. 7, p. 278, 2016.

[207] N. S. Gooneratne, A. Y. Z. Edwards, C. Zhou, N. Cuellar, M. A. Grandner, and J. S. Barrett, "Melatonin pharmacokinetics following two different oral surge-sustained release doses in older adults," Journal of Pineal Research, vol. 52, no. 4, pp. 437-445, 2012.

[208] M. Vermeulen, I. W. A. A. Klöpping-Ketelaars, R. Van Den Berg, and W. H. J. Vaes, "Bioavailability and kinetics of sulforaphane in humans after consumption of cooked versus raw broccoli," Journal of Agricultural and Food Chemistry, vol. 56, no. 22, pp. 10505-10509, 2008.

[209] S. Mishra and K. Palanivelu, "The effect of curcumin (turmeric) on Alzheimer's disease: an overview," Annals of Indian Academy of Neurology, vol. 11, no. 1, pp. 13-19, 2008.

[210] B. Grimmig, S. H. Kim, K. Nash, P. C. Bickford, and R. Douglas Shytle, "Neuroprotective mechanisms of astaxanthin: a potential therapeutic role in preserving cognitive function in age and neurodegeneration," GeroScience, vol. 39, no. 1, pp. 19-32, 2017.

[211] M. Qu, L. Li, C. Chen et al., "Protective effects of lycopene against amyloid $\beta$-induced neurotoxicity in cultured rat cortical neurons," Neuroscience Letters, vol. 505, no. 3, pp. 286-290, 2011.

[212] J. N. Saykally, L. Rachmany, H. Hatic et al., "The nuclear factor erythroid 2-like 2 activator, tert-butylhydroquinone, improves cognitive performance in mice after mild traumatic brain injury," Neuroscience, vol. 223, pp. 305-314, 2012.
[213] N. H. R. Litjens, J. Burggraaf, E. van Strijen et al., "Pharmacokinetics of oral fumarates in healthy subjects," British Journal of Clinical Pharmacology, vol. 58, no. 4, pp. 429-432, 2004.

[214] M. A. Seifman, A. A. Adamides, P. N. Nguyen et al., "Endogenous melatonin increases in cerebrospinal fluid of patients after severe traumatic brain injury and correlates with oxidative stress and metabolic disarray," Journal of Cerebral Blood Flow \& Metabolism, vol. 28, no. 4, pp. 684-696, 2008.

[215] F. Zhang, J. Xing, A. K. F. Liou et al., "Enhanced delivery of erythropoietin across the blood-brain barrier for neuroprotection against ischemic neuronal injury," Translational Stroke Research, vol. 1, no. 2, pp. 113-121, 2010.

[216] E. Burgos-Morón, J. M. Calderón-Montaño, J. Salvador, A. Robles, and M. López-Lázaro, "The dark side of curcumin," International Journal of Cancer, vol. 126, pp. 17711775, 2010.

[217] A. Satoh, S. Tsuji, Y. Okada et al., "Preliminary clinical evaluation of toxicity and efficacy of a new astaxanthin-rich Haematococcus pluvialis extract," Journal of Clinical Biochemistry and Nutrition, vol. 44, no. 3, pp. 280-284, 2009.

[218] G. J. van Esch, "Toxicology of tert-butylhydroquinone (TBHQ)," Food and Chemical Toxicology, vol. 24, no. 10-11, pp. 1063-1065, 1986.

[219] "Dimethyl fumarate toxicity," https://toxnet.nlm.nih.gov/cgibin/sis/search/a?dbs+hsdb:@term+@DOCNO+7725.

[220] B. Guardiola-Lemaitre, "Toxicology of melatonin," Journal of Biological Rhythms, vol. 12, no. 6, pp. 697-706, 1997. 


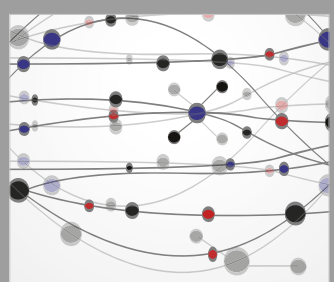

The Scientific World Journal
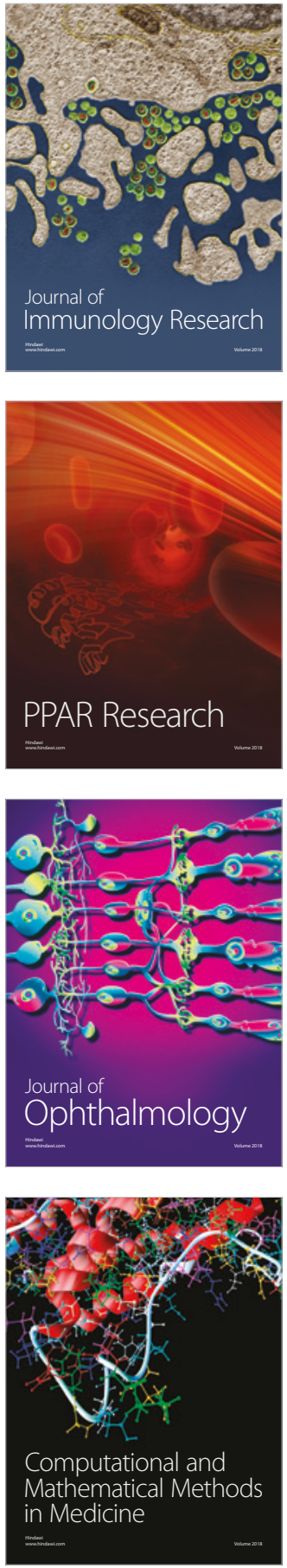

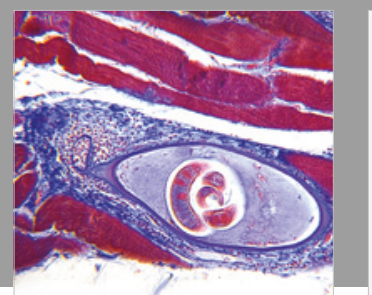

Gastroenterology Research and Practice

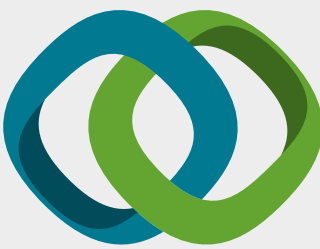

\section{Hindawi}

Submit your manuscripts at

www.hindawi.com
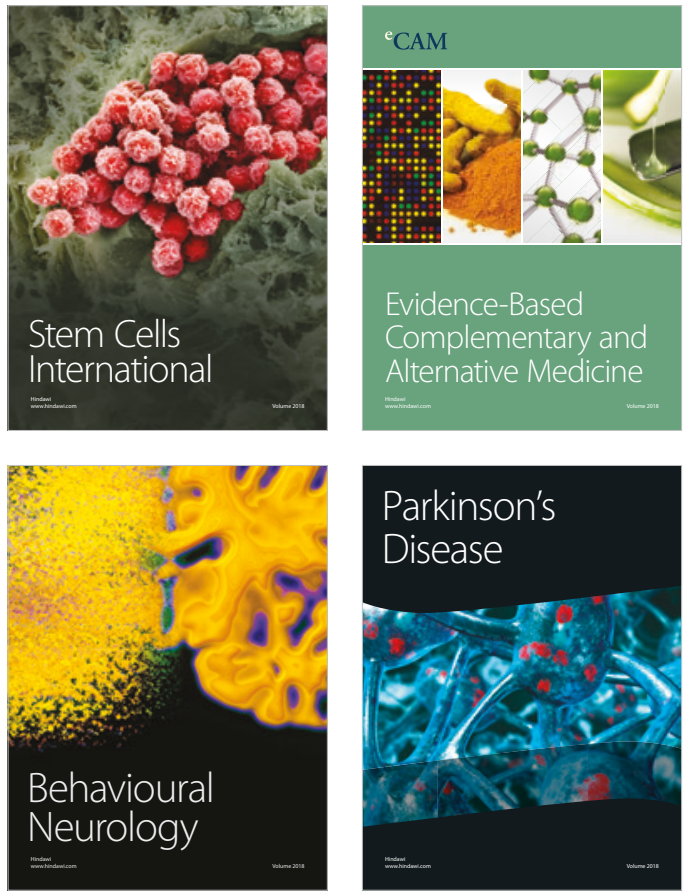

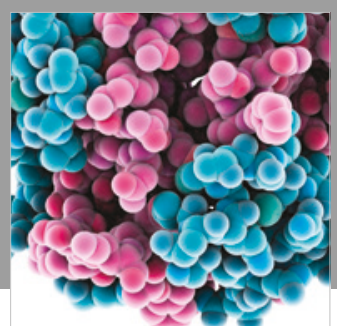

ournal of

Diabetes Research

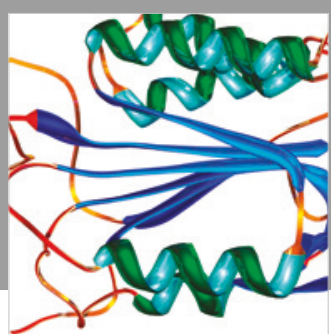

Disease Markers
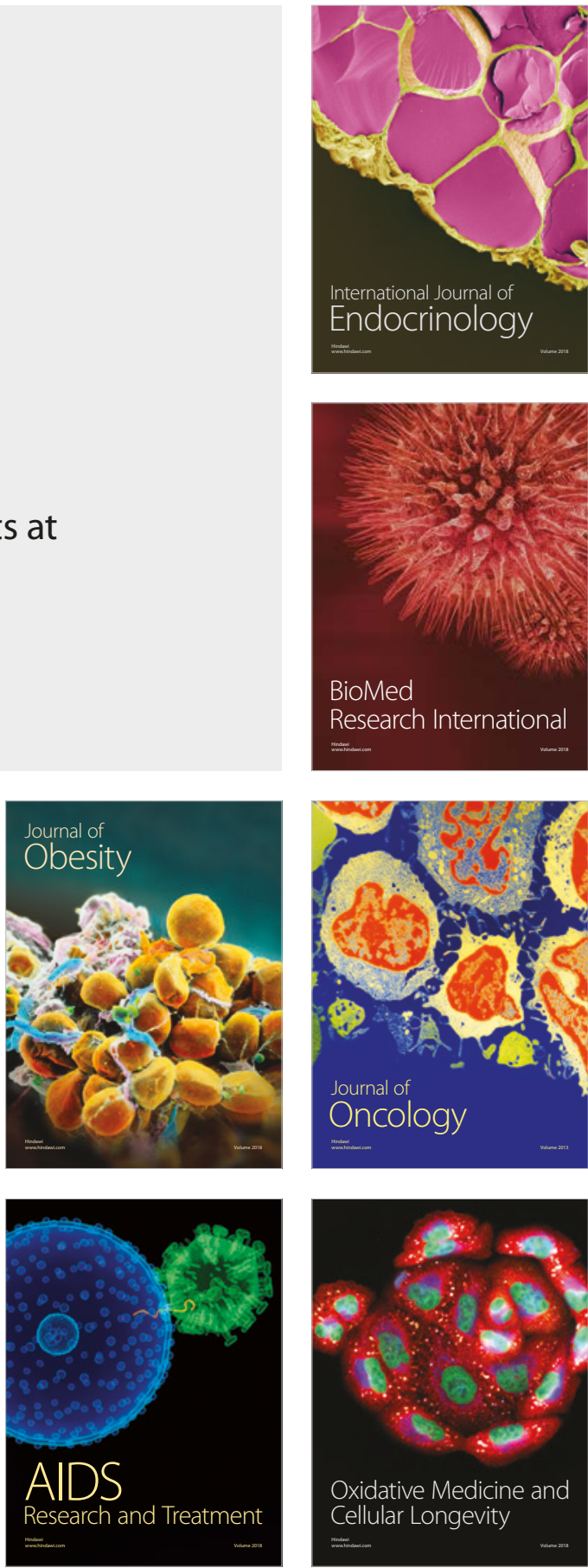